\title{
THE UNSTEADY BOUNDARY LAYER ON A CYLINDER DUE TO SMALL TRANSVERSE OSCILLATIONS*
}

\author{
BY \\ D. E. WILSON \\ University of Texas at Austin
}

\begin{abstract}
This paper deals with the response of an incompressible laminar boundary layer on a circular cylinder due to small periodic velocity fluctuations. These fluctuations may arise from the motion of the cylinder or be contained within the free stream. A perturbation solution employing the method of matched asymptotic expansions is given for the case of high reduced frequencies. These results are compared to a numerical solution valid for any frequency. In addition, the solution is compared to the classical result of Glauert [7] valid only at the stagnation point.
\end{abstract}

1. Introduction. In unsteady flow, potential theory has been used quite successfully to describe many important aerodynamic characteristics. However, the inviscid approach is inadequate for describing detailed features and basic mechanisms present in many unsteady flows. In these cases, time-dependent boundary-layer theory must be employed to describe a number of unsteady viscous effects such as temporal and spatial phase differences, nonlinear streaming, separation delay and viscous damping. A physical description of the phenomena which characterize unsteady boundary layers and the related mathematical analysis can be found in the excellent review articles of Riley [12] and McCroskey [11].

Most theoretical studies of unsteady boundary layers have been concerned with the response of laminar incompressible boundary layers to small periodic fluctuations in the free stream. The first substantial efforts were due to Lighthill [9] and Lin [10]. With few exceptions, the analysis has concentrated on the flow past a semi-infinite flat plate or in the vicinity of a two-dimensional stagnation point. Among the first rigorous treatments, which demonstrated the asymptotic structure of the unsteady boundary layer, is the analysis of Ackerberg and Phillips [1].

\footnotetext{
${ }^{*}$ Received November 4, 1985.
} 
When the free stream approaches normal to an infinite flat plate with oscillations in its own plane, the Navier-Stokes equations admit an "exact" solution. This was first recognized independently in the seminal papers of Glauert [7] and Rott [13]. The infinite and planar geometry allows the flow to be split into a steady and unsteady part, which is dependent only on the normal coordinate and time. Subsequent investigations of stagnation point oscillations appear to be influenced by this feature in seeking solutions in the neighborhood of a stagnation point. For example, Ishigaki [8] assumes an imposed velocity distribution of the form

$$
U_{e}(x, t)=A x\left(1+\varepsilon e^{i \omega t}\right)
$$

Ghoshal and Ghoshal [6] use a similar assumption for a three-dimensional stagnation point. Although the mathematical analysis is sound, this distribution is not physically realistic for transverse oscillations in the neighborhood of a bluff body.

The purpose of this paper is to investigate the behavior of an incompressible laminar boundary layer on a circular cylinder subjected to small periodic fluctuations. The formulation provides the flexibility to model arbitrary oscillations of the free stream and cylinder; however, results are presented only for the case of transverse oscillations.

The solution is accomplished by first expanding the stream function in terms of $\varepsilon$, where $\varepsilon$ is the ratio of the transverse velocity component to the undisturbed free stream velocity. The resulting equations are then solved by an asymptotic expansion in terms of the reduced frequency. Due to the singular nature of the perturbation problem, an inner and outer region are defined and the method of matched asymptotic expansions is employed. The result is compared to a numerical solution valid for all frequencies.

The analysis is intended to represent the unsteady flow over a cylinder, based upon the following implicit assumptions. First, the influence of fluctuations inherent in the unsteady wake must be small compared to the influence of the imposed oscillation. This interaction between the wake and the inviscid flow, which is usually ignored, was studied by Dwyer and McCroskey [11]. Their investigation of these self-induced oscillations, at a $R e=1.06 \times 10^{5}$, shows only a small deviation of wall shear stress in the neighborhood of the stagnation point. The second assumption, which is consistent with the mathematical restrictions, is that the imposed oscillation does not significantly alter the wake. It is well known that an oscillating cylinder can control the instability mechanism which leads to the vortex shedding. However, this effect, as discussed by Bearman [2], is not significant for small amplitude oscillations which are consistent with the restrictions of the perturbation analysis.

2. Mathematical formulation. The problem considered in this investigation is that of an unsteady incompressible flow over an infinite circular cylinder. The unsteady component may originate from free stream or cylinder oscillations provided that the unsteady velocity is small when compared to the free stream velocity.

For the case of an infinite cylinder, the flow will be two-dimensional. The usual boundary-layer coordinates are adopted, where $x$ denotes the coordinate along the surface and $y$ is the coordinate normal to the surface. Curvature can be neglected in the analysis through first order as discussed by Gersten [5] and Van Dyke [15]. 
Defining the stream function, $\psi$, as

$$
u=\frac{\partial \psi}{\partial y} \quad \text { and } \quad v=-\frac{\partial \psi}{\partial x}
$$

allows the boundary layer equations to be written as

$$
\frac{\partial^{2} \psi}{\partial y \partial t}+\frac{\partial \psi}{\partial y} \frac{\partial^{2} \psi}{\partial x \partial y}-\frac{\partial \psi}{\partial x} \frac{\partial^{2} \psi}{\partial y^{2}}=\frac{\partial U_{e}}{\partial t}+U_{e} \frac{\partial U_{e}}{\partial x}+\frac{\partial^{3} \psi}{\partial y^{3}} .
$$

The boundary conditions are simply

$$
\frac{\partial \psi}{\partial x}=\frac{\partial \psi}{\partial y}=0 \quad \text { at } \quad y=0
$$

and

$$
\frac{\partial \psi}{\partial y} \rightarrow U_{e}(x, t) \text { as } \quad y \rightarrow \infty
$$

In Equations (1) and (3), $U_{e}$ represents the velocity at the edge of the boundary layer which will be determined from potential theory.

Outside the boundary layer, the flow is irrotational and the equation of motion is simply

$$
\nabla^{2} \Psi=0
$$

The surface boundary condition for the case of a cylinder undergoing arbitrary translations, as shown in Fig. 1, is given by

$$
v_{r}(R, \theta)=-\left.\frac{1}{r} \frac{\partial \Psi}{\partial \theta}\right|_{R}=\dot{X}_{0} \operatorname{Cos} \theta+\dot{Y}_{0} \operatorname{Sin} \theta
$$

The stream function with respect to the laboratory system then becomes

$$
\Psi(r, \theta)=\frac{R^{2} \dot{Y}_{0}}{r} \operatorname{Cos} \theta-\frac{R^{2} \dot{X}_{0}}{r} \operatorname{Sin} \theta-U_{\infty}\left(r-\frac{R^{2}}{r}\right) \operatorname{Sin} \theta .
$$

Using (6), the velocity on the surface in terms of boundary layer coordinates can be readily obtained. Taking the coordinate system fixed on the cylinder and considering only vertical translations yields

$$
U_{e}(x, t)=z U_{\infty} \operatorname{Sin}(x / R)-2 V_{0} \operatorname{Cos}(x / R) \operatorname{Cos} \omega t .
$$

For the second case of a transversely oscillating free stream approaching a fixed cylinder, the edge velocity is also given by

$$
U_{e}(x, t)=2 U_{\infty} \operatorname{Sin}(x / R)-2 V_{0} \operatorname{Cos}(x / R) \operatorname{Cos} \omega t .
$$

This flow is representative of a wind tunnel with periodic transpiration at the walls. The actual flow is somewhat complicated and depends upon the transpiration length, tunnel height, and body geometry. 


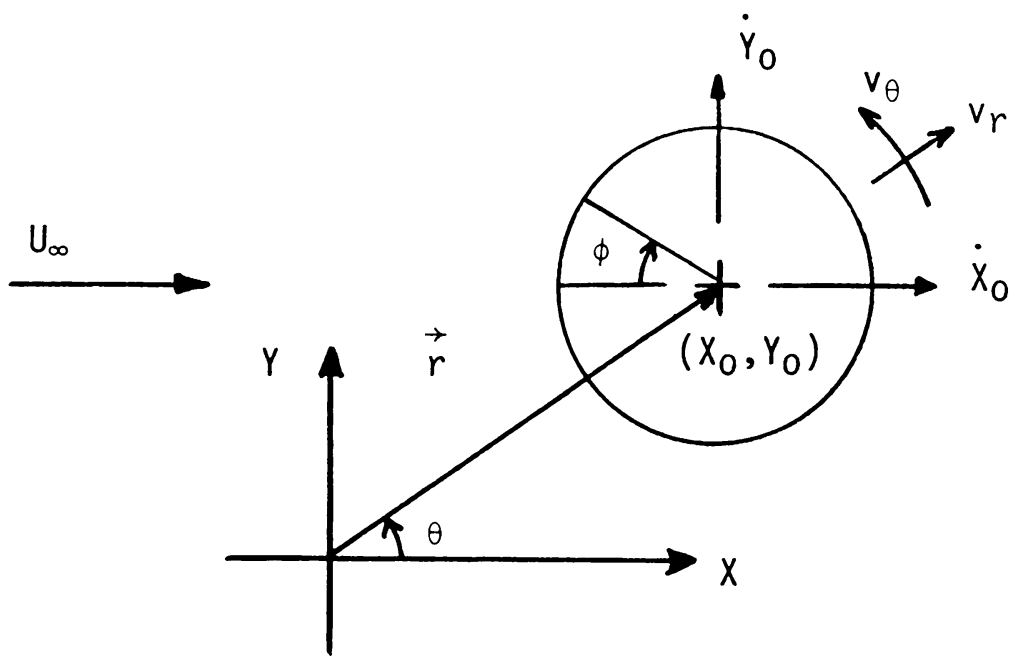

FIG. 1. Oscillating cylinder in a uniform flow.

2.1. Nondimensional equations. The flow in the boundary layer is characterized by two different viscous length scales. They are denoted by $\delta_{s}$ and $\delta_{B}$, where $\delta_{s} \sim(\nu / \omega)^{1 / 2}$ is the unsteady Stokes layer and $\delta_{B} \sim(\nu / c)^{1 / 2}$ is the steady boundary layer or Heimenz layer. The parameter, $c$, represents the characteristic convection time scale given by $4 V_{\infty} / D$. The Stokes layer is a measure of diffusion due to the fluctuating component of the flow. When a steady mean flow is present and $\omega / c=O(1)$, the two layers are of comparable thickness and the resulting flow is quite complex. However, when the frequency is "large" such that $\delta_{s} / \delta_{B} \ll 1$, the fluctuations are confined to a thin layer near the wall. This feature is exploited in the perturbation analysis to produce a significant simplification in the resulting equations. With this in mind, the initial set of nondimensional variables and parameters are defined below:

$$
\begin{aligned}
\eta & \equiv \frac{y}{(\nu / c)^{1 / 2}}, \quad \zeta \equiv \frac{x}{R}, \quad \tau \equiv \omega t \\
\hat{\psi} & \equiv \frac{\psi}{\left(2 \nu U_{\infty} R\right)^{1 / 2}}, \quad \hat{U} \equiv \frac{U}{2 U_{\infty}}, \quad \hat{v} \equiv \frac{v}{2 U_{\infty}} R e^{1 / 2} \\
\operatorname{Re} & \equiv \frac{U_{\infty} D}{\nu}, \quad \alpha \equiv \frac{\omega D}{4 U_{\infty}}, \quad \varepsilon \equiv \frac{V_{0}}{U_{0}}
\end{aligned}
$$

Substituting (9) into (1) through (3) yields

$$
\alpha \frac{\partial^{2} \hat{\psi}}{\partial \eta \partial t}+\frac{\partial \hat{\psi}}{\partial \eta} \frac{\partial^{2} \hat{\psi}}{\partial \eta \partial \zeta}-\frac{\partial \hat{\psi}}{\partial \zeta} \frac{\partial^{2} \hat{\psi}}{\partial \eta^{2}}-\frac{\partial^{3} \hat{\psi}}{\partial \eta^{3}}=\alpha \frac{\partial \hat{U}_{e}}{\partial \tau}+U_{e} \frac{\partial \hat{U}_{e}}{\partial \zeta}
$$

with boundary conditions given by

$$
\frac{\partial \hat{\psi}}{\partial \eta}=\frac{\partial \hat{\psi}}{\partial \zeta}=0 \quad \text { at } \quad \eta=0
$$


and

$$
\frac{\partial \hat{\psi}}{\partial \eta} \rightarrow \hat{U}_{e}(\zeta, \tau) \text { as } \quad \eta \rightarrow \infty .
$$

The velocity at the edge of the boundary layer for the transversely oscillating cylinder becomes

$$
\hat{U}_{e}(\zeta, \tau)=\operatorname{Sin} \zeta-\varepsilon \operatorname{Cos} \zeta \operatorname{Cos} \tau
$$

The two nondimensional parameters which explicitly appear in the boundary layer equations characterize the unsteady flow. The perturbation parameter, $\varepsilon$, is a measure of the unsteadiness, and the reduced frequency, $\alpha$, is a measure of the steady to unsteady diffusion of vorticity in the boundary layer.

2.2. Small fluctuation equations. A solution to (10)-(12) is sought for situations when the transverse oscillations of the cylinder or free stream are small with respect to $U_{\infty}$. This ratio, which appears in (13), is taken as the perturbation parameter. The edge velocity can then be written as

$$
\hat{U}_{e}(\zeta, \tau)=\hat{U}_{0}(\zeta)+\varepsilon \hat{U}_{1}(\zeta, \tau)
$$

where $\hat{U}_{1}(\zeta, \tau)$ represents the general unsteady component. Writing the stream function as

$$
\hat{\psi}(\zeta, \eta, \tau ; \alpha, \varepsilon)=\hat{\Psi}_{0}(\zeta, \eta)+\varepsilon \hat{\psi}_{1}(\zeta, \eta, \tau ; \alpha)+O\left(\varepsilon^{2}\right)
$$

and substituting into (10)-(12) yields the following system of equations: ${ }^{1}$

$$
\begin{gathered}
\frac{\partial \hat{\psi}_{0}}{\partial \eta} \frac{\partial^{2} \hat{\psi}_{0}}{\partial \zeta \partial \eta}-\frac{\partial \hat{\psi}_{0}}{\partial \zeta} \frac{\partial^{2} \hat{\psi}_{0}}{\partial \eta^{2}}-\frac{\partial^{3} \hat{\psi}_{0}}{\partial \eta^{3}}=\hat{U}_{0} \frac{d \hat{U}_{0}}{d \zeta} \\
\alpha \frac{\partial^{2} \hat{\psi}_{1}}{\partial \eta \partial \tau}+\hat{\psi}_{0, \eta} \frac{\partial^{2} \hat{\psi}_{1}}{\partial \zeta \partial \eta}+\hat{\psi}_{0, \zeta \eta} \frac{\partial \hat{\psi}_{1}}{\partial \eta}-\psi_{0, \zeta} \frac{\partial^{2} \hat{\psi}_{1}}{\partial \eta^{2}}-\hat{\psi}_{0, \eta \eta} \frac{\partial \hat{\psi}_{1}}{\partial \zeta}-\frac{\partial^{3} \hat{\psi}_{1}}{\partial \eta^{3}} \\
=\alpha \frac{\partial \tilde{U}_{1}}{\partial \tau}+\hat{U}_{0} \frac{\partial \hat{U}_{1}}{\partial \zeta}+\hat{U}_{1} \frac{d \hat{U}_{0}}{d \zeta}
\end{gathered}
$$

When $\varepsilon=0$, the problem reduces to steady uniform flow past a circular cylinder as expected, subject to the following boundary conditions:

$$
\begin{aligned}
& \frac{\partial \hat{\psi}_{0}}{\partial \eta}=\frac{\partial \hat{\psi}_{0}}{\partial \zeta}=0 \quad \text { at } \quad \eta=0, \\
& \frac{\partial \hat{\psi}_{0}}{\partial \eta} \rightarrow \hat{U}_{0}(\zeta) \text { as } \eta \rightarrow \infty
\end{aligned}
$$

\footnotetext{
${ }^{1}$ The notation $\psi_{0, \eta}$ denotes differentiation with respect to $\eta$, etc.
} 
The fluctuating component $\psi_{1}$ can then be found by solving (17) subject to

$$
\begin{gathered}
\frac{\partial \hat{\psi}_{1}}{\partial \eta}=\frac{\partial \hat{\psi}_{1}}{\partial \zeta}=0 \quad \text { at } \quad \eta=0, \\
\frac{\partial \hat{\psi}_{1}}{\partial \eta} \rightarrow \hat{U}_{1}(\zeta, \tau) \quad \text { as } \quad \eta \rightarrow \infty .
\end{gathered}
$$

Before proceeding, it is important to discuss the limitations inherent in the potential flow/boundary-layer formulation and how they relate to the unsteady perturbation analysis.

To insure that the predominant perturbation to the flow is due to the unsteady fluctuations and not higher-order boundary-layer effects, some constraint on the steady Reynolds number must be given. The two important second-order effects are due to the displacement thickness and curvature. Applying the method of matched asymptotic expansions to the Navier-Stokes equations, it can be shown, Gersten [5], that both curvature and displacement effects are $O\left(R e^{-1 / 2}\right)$ relative to the first-order boundary-layer theory. Therefore, we must require that $\varepsilon \gg R e^{-1 / 2}$, which does not pose any serious limitation for realistic values of the Reynolds number. However, this second-order curvature effect applies only for steady flow and should be modified accordingly for unsteady flows. Writing the inviscid normal pressure gradient as

$$
\frac{1}{\rho} \frac{\partial p}{\partial n}=\frac{V^{2}}{R}+V \frac{\partial \theta}{\partial t}
$$

and nondimensionalizing, yields

$$
\frac{\partial \bar{p}}{\partial n}=\frac{1}{R e^{1 / 2}}\left(\bar{V}^{2}+\alpha \varepsilon \bar{V} \frac{\partial \theta}{\partial \tau}\right)
$$

This condition implies that $\alpha \varepsilon<1$ or that the upper bound of the reduced frequency be limited by $\alpha=O(1 / \varepsilon)$.

3. High frequency solutions. In this section, a solution to (17) valid for high reduced frequencies is obtained. Formally, this implies that $\alpha>1$. However, for (17) to be valid, we must also required $a / D=O(\varepsilon)$, where $a$ is the oscillation amplitude. This insures that the unsteady pressure term in (17) be of proper order, and implies that the amplitude of oscillation be inversely proportional to the frequency. Under these conditions, a straightforward asymptotic expansion in terms of $1 / \alpha$ leads to the following equation describing the leading behavior:

$$
\frac{\partial^{2} \hat{\psi}_{1}}{\partial \eta \partial \tau}=\frac{\partial \hat{U}_{1}}{\partial \tau}
$$

The solution, subject to Equation (19), is

$$
\hat{\psi}_{1}=(C(\zeta)-\eta \operatorname{Cos} \zeta) e^{i \tau}
$$

and it is obvious that the boundary condition at the wall cannot be satisfied. This is a classical hallmark of singular perturbation problems and is due mathematically to a reduction in the order of the differential equation. Physically, the situation is explained by 
the existence of a thin layer near the wall where the viscous effects are concentrated and the major part of the boundary layer reacts as if it were effectively inviscid.

The problem is readily resolved by employing a matched asymptotic expansion. The equations, which are properly scaled to represent the physics in the outer region, are solved subject to the boundary conditions at the edge of the boundary layer. In the inner region the equations must be rewritten with respect to the new inner variables. This solution is then matched with the outer solution to form a composite solution. A sketch of the inner and outer region is given in Fig. 2. Also shown in this figure is the region about the mean stagnation point for which the analysis is valid.

Before proceeding with the high-frequency expansion, a final and somewhat subtle point should be made. It is known, Stuart [14], that in the absence of an external flow, an oscillating cylinder induces a steady streaming motion, the magnitude of which is proportional to $V_{0}^{2} /(\omega D)$. In order that the high-frequency expansion remain valid in comparison to the imposed oscillation requires that $V_{0} /(\omega D) \ll 1$, or simply that $a / D \ll$ 1. This requirement has already been stated and thus introduces no additional restriction.

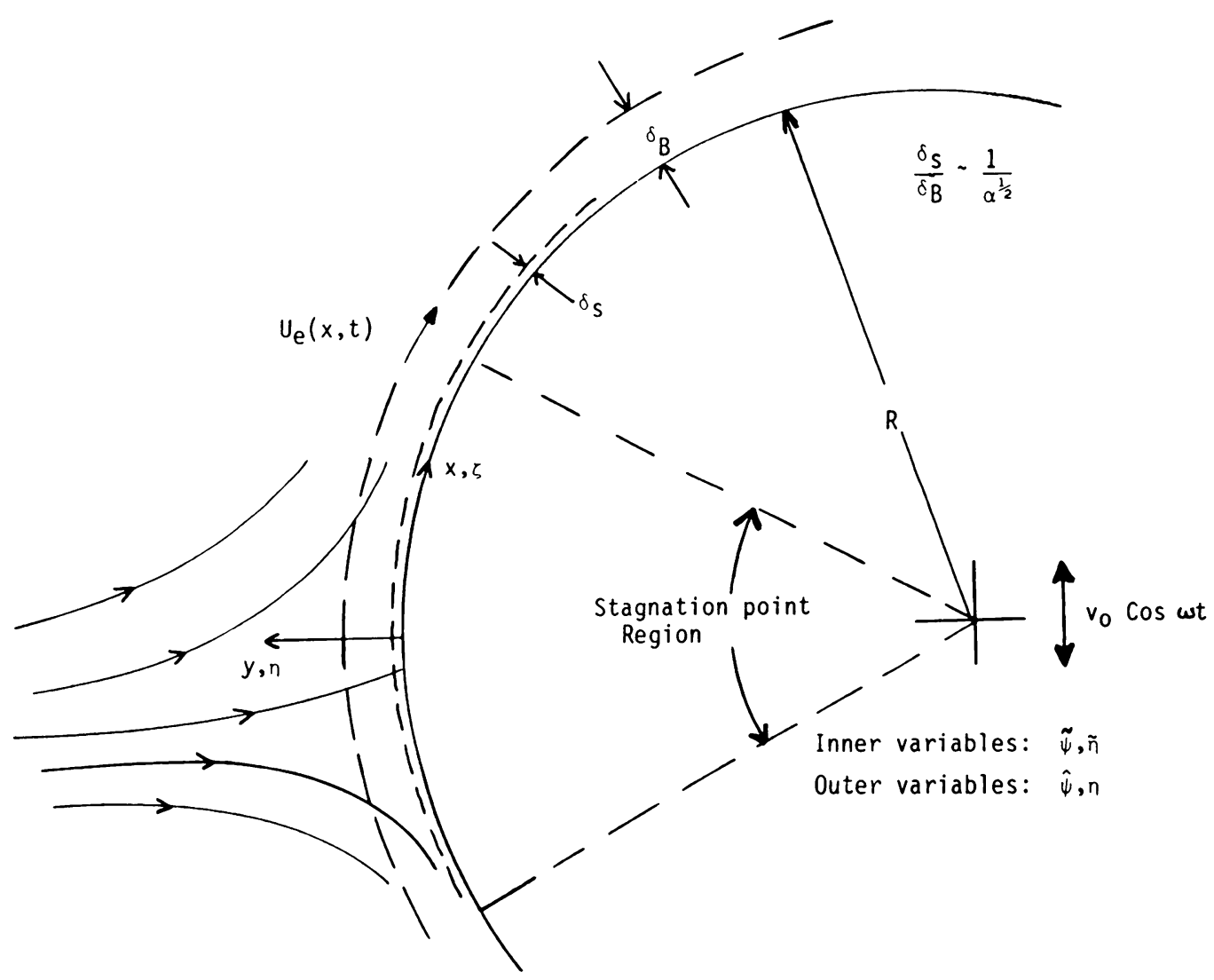

FIG. 2. Flow structure and coordinates 
4.1. Outer solution. The nonhomogeneous term in (17) implies that $\hat{\psi}_{1}(\zeta, \eta, \tau)$ can be written as

$$
\hat{\psi}_{1}(\zeta, \eta, \tau)=\hat{\chi}(\zeta, \eta) e^{i \tau} .
$$

Using (20) in (17) yields the following equation

$$
\begin{array}{r}
\frac{\partial \hat{\chi}}{\partial \eta}=\operatorname{Cos} \zeta-i \delta^{2}\left\{-\operatorname{Cos} 2 \zeta+\hat{\psi}_{0, \zeta} \frac{\partial^{2} \hat{\chi}}{\partial \eta^{2}}+\hat{\psi}_{0, \eta \eta} \frac{\partial \hat{\chi}}{\partial \zeta}\right. \\
\left.-\hat{\psi}_{0, \eta} \frac{\partial \hat{\chi}}{\partial \zeta \partial \eta}-\hat{\psi}_{0, \zeta \eta} \frac{\partial \hat{\chi}}{\partial \eta}+\frac{\partial^{3} \hat{\chi}}{\partial \eta^{3}}\right\} .
\end{array}
$$

Similarly, the boundary conditions can be written as

$$
\frac{\partial \hat{\chi}}{\partial \eta}=\frac{\partial \hat{\chi}}{\partial \zeta}=0 \quad \text { at } \quad \eta=0
$$

and

$$
\frac{\partial \hat{\chi}}{\partial \eta} \rightarrow \operatorname{Cos} \zeta \quad \text { as } \quad \eta \rightarrow \infty
$$

In these equations, the unsteady velocity term, $\hat{U}_{1}$, has been taken to represent the case of a transversely oscillating cylinder. Also, in (21), the parameter, $\delta$, has been introduced; it represents the ratio of the Stokes layer to the Hiemenz layer and is defined as

$$
\delta \equiv \alpha^{-1 / 2}=\left(\frac{(\nu / \omega)}{\nu / c}\right)^{1 / 2}
$$

Expanding $\hat{\chi}(\zeta, \eta)$ in terms of $\delta$ according to

$$
\hat{\chi}(\zeta, \eta)=\sum_{n=0}^{N} \delta^{n} \phi_{n}(\zeta, \eta)+O\left(\delta^{n+1}\right)
$$

and then substituting into (21) yields the following equations for $\phi_{n}$ :

$$
\begin{aligned}
& \frac{\partial \phi_{0}}{\partial \eta}=-\operatorname{Cos} \zeta, \\
& \frac{\partial \phi_{1}}{\partial \eta}=0, \\
& \frac{\partial \phi_{2}}{\partial \eta}=-i\left\{-\operatorname{Cos} 2 \zeta+\hat{\psi}_{0, \zeta} \frac{\partial^{2} \phi_{0}}{\partial \eta^{2}}+\hat{\psi}_{0, \eta \eta} \frac{\partial \phi_{0}}{\partial \zeta}\right. \\
& \left.-\hat{\psi}_{0, \eta} \frac{\partial^{2} \phi_{0}}{\partial \zeta \partial \eta}-\hat{\psi}_{0, \xi \eta} \frac{\partial \phi_{0}}{\partial \eta}+\frac{\partial^{3} \phi_{0}}{\partial \eta^{3}}\right\},
\end{aligned}
$$




$$
\begin{array}{r}
\frac{\partial \phi_{n}}{\partial \eta}=-i\left\{\hat{\psi}_{0, \zeta} \frac{\partial^{2} \phi_{n-2}}{\partial \eta^{2}}+\hat{\psi}_{0, \eta \eta} \frac{\partial \phi_{n-2}}{\partial \zeta}-\hat{\psi}_{0, \eta} \frac{\partial^{2} \phi_{n-2}}{\partial \zeta \partial \eta}\right. \\
\left.-\hat{\psi}_{0, s \eta} \frac{\partial \phi_{n-2}}{\partial \eta}+\frac{\partial^{3} \phi_{n-2}}{\partial \eta^{3}}\right\} .
\end{array}
$$

These equations are subject to the outer boundary conditions given below:

$$
\frac{\partial \phi_{0}}{\partial \eta} \rightarrow-\operatorname{Cos} \zeta \quad \text { as } \quad \eta \rightarrow \infty
$$

and

$$
\frac{\partial \phi}{\partial \eta} \rightarrow 0 \quad \text { as } \quad \eta \rightarrow \infty
$$

The solutions to the first two equations which clearly satisfy the outer boundary condition are given by

$$
\phi_{0}=-\eta \operatorname{Cos} \zeta+C_{0}(\zeta)
$$

and

$$
\phi_{1}=C_{1}(\zeta)
$$

The unknown functions of integration, i.e., $C_{n}(\zeta)$, must be found by matching with the inner solution. Using these two solutions, the equations for $\phi_{2}$ and $\phi_{3}$ become

$$
\begin{aligned}
\frac{\partial \phi_{2}}{\partial \eta}=i\left\{\operatorname { S i n } \zeta \left(\hat{\psi}_{0, \eta}-\right.\right. & \left.\eta \hat{\psi}_{0, \eta \eta}\right)-\hat{\psi}_{0, \zeta \eta} \operatorname{Cos} \zeta \\
& \left.+\operatorname{Cos} 2 \zeta+C_{0}^{\prime}(\zeta) \hat{\psi}_{0, \eta \eta}\right\}
\end{aligned}
$$

and

$$
\frac{\partial \phi_{3}}{\partial \eta}=-i\left\{\hat{\psi}_{0, \eta \eta} C_{1}^{\prime}(\zeta)\right\}
$$

The solutions are then

$$
\begin{aligned}
\phi_{2}=i\left\{\operatorname{Sin} \zeta\left(2 \hat{\psi}_{0}-\eta \hat{\psi}_{0, \eta}\right)-\hat{\psi}_{0, \zeta} \operatorname{Cos} \zeta\right. \\
\left.+\eta \operatorname{Cos} 2 \zeta+C_{2}(\zeta)+\hat{\psi}_{0, \eta} C_{0}^{\prime}(\zeta)\right\}
\end{aligned}
$$

and

$$
\phi_{3}=-i \hat{\psi}_{0, \eta} C_{1}^{\prime}(\zeta)+C_{3}(\zeta) .
$$

The fact that these solutions also satisfy the outer boundary condition can be shown by noting that $\hat{\psi}_{0, \eta} \rightarrow \operatorname{Sin} \zeta, \hat{\psi}_{0, \eta \eta} \rightarrow 0$, and $\hat{\psi}_{0, \zeta \eta} \rightarrow \operatorname{Cos} \zeta$ as $\eta \rightarrow \infty$.

Higher-order solutions are conceptually straightforward; however, they become quite lengthy and in general must be written in terms of closed form integral expressions. For $n \geqslant 4$, the solution for the stream function becomes

$$
\phi_{n}=-i\left\{\hat{\psi}_{0, \eta} \phi_{\zeta}+\hat{\psi}_{0, \zeta} \phi_{\eta}+\phi_{\eta \eta}-2 \frac{\partial}{\partial \zeta} \int \hat{\psi}_{0, \eta} \phi_{\eta} d \eta\right\}_{n-2} .
$$


Complete solutions in terms of the stream function are given only to $O\left(\delta^{4}\right)$. However, solutions for the $\zeta$-component of velocity are found to $O\left(\delta^{6}\right)$ in Sec. 4.3.

4.2. Inner solution. In the inner region, it is important that both the stream function and the normal coordinate be stretched. Using the Stokes layer as the appropriate scale, the following nondimensional variables are defined:

$$
\begin{aligned}
& \tilde{\eta} \equiv \frac{y}{(\nu / \omega)^{1 / 2}}=\eta / \delta, \quad \tilde{\zeta} \equiv \zeta, \\
& \tilde{\psi} \equiv \frac{\psi}{2 U_{\infty}(\nu / \omega)^{1 / 2}}=\hat{\psi} / \delta, \quad \tilde{\tau} \equiv \tau .
\end{aligned}
$$

Using these variables, (17) is transformed to the following equation valid in the inner region.

$$
\begin{aligned}
\frac{\partial^{2} \tilde{\psi}_{1}}{\partial \tilde{\eta} \partial \tau}-\frac{\partial^{3} \tilde{\psi}_{1}}{\partial \tilde{\eta}^{3}}-\frac{\partial \hat{U}_{1}}{\partial \tau} & =\delta\left\{\hat{\psi}_{0, \zeta} \frac{\partial^{2} \tilde{\psi}_{1}}{\partial \tilde{\eta}^{2}}\right\} \\
& +\delta^{2}\left\{\hat{U}_{0} \frac{\partial \hat{U}_{0}}{\partial \zeta}+\hat{U}_{1} \frac{\partial \hat{U}_{0}}{\partial \zeta}-\hat{\psi}_{0, \eta} \frac{\partial^{2} \tilde{\psi}_{1}}{\partial \zeta \partial \tilde{\eta}}-\hat{\psi}_{0, \zeta \eta} \frac{\partial \tilde{\psi}_{1}}{\partial \tilde{\eta}}\right\}+\delta^{3}\left\{\hat{\psi}_{0, \eta \eta} \frac{\partial \tilde{\psi}_{1}}{\partial \zeta}\right\}
\end{aligned}
$$

As before, the stream function may be written as

$$
\tilde{\psi}_{1}(\zeta, \eta, \tau)=\hat{\chi}(\zeta, \tilde{\eta}) e^{i \tau}
$$

and $\tilde{\chi}_{1}(\zeta, \eta)$ can be expanded in terms of $\delta$ as

$$
\tilde{\chi}(\zeta, \tilde{\eta})=\sum_{n=0}^{N} \delta^{n} \Phi_{n}(\zeta, \tilde{\eta})+O\left(\delta^{N+1}\right) .
$$

However, before these results are substituted into Equation (39), the coefficients, $\hat{\psi}_{0,5}$, etc., must be rewritten in terms of the inner variables. In doing this, it is important to remember that the inner solution is obtained by letting $\delta \rightarrow 0$ with $\eta$ fixed, but the coefficients are functions of $\eta$ where $\eta=\delta \tilde{\eta}$. Therefore, the coefficients should be expanded for small $\eta$ and rewritten in terms of $\delta \tilde{\eta}$. This procedure is outlined next.

The steady state solution, $\psi_{0}$, is first expanded using the Howarth series as

$$
\hat{\psi}_{0}(\zeta, \eta)=f_{0}(\eta) \zeta+a_{2} f_{2}(\eta) \zeta^{3}+\left(a_{4} f_{4}(\eta)+a_{2}^{2} f_{22}(\eta)\right) \zeta^{5}+\cdots .
$$

This expansion, when substituted into (16), produces a series of coupled boundary value problems given by (A.3) through (A.11). A local expansion near $\eta=0$ is then determined from these equations. The result for the stagnation point is well known and is given by

$$
f_{0}(\eta)=\beta_{0} \eta^{2} / 2 !-\eta^{3} / 3 !+\beta_{0}^{2} \eta^{5} / 5 !+O\left(\eta^{6}\right) .
$$

The local behavior for the remaining equations must be generated by a series expansion in powers of $\eta$. This series is substituted into each of the remaining equations, (A.4)-(A.11). 
Then using the boundary condition at $\eta=0$, the constants are determined. This straightforward but lengthy procedure yields the following results through $O\left(\eta^{6}\right)$ :

$$
\begin{aligned}
f_{2}(\eta) & =\beta_{2} \eta^{2} / 2 !-4 \eta^{3} / 3 !+\beta_{0} \beta_{2} \eta^{5} / 5 !+O\left(\eta^{6}\right), \\
f_{4}(\eta) & =\beta_{4} \eta^{2} / 2 !-6 \eta^{3} / 3 !+\beta_{0} \beta_{4} \eta^{5} / 5 !+O\left(\eta^{6}\right), \\
f_{22}(\eta) & =\beta_{22} \eta^{2} / 2 !-3 \eta^{3} / 3 !+\left(\beta_{0} \beta_{22}+3 \beta_{2}^{2}\right) \eta^{5} / 5 !+O\left(\eta^{6}\right),
\end{aligned}
$$

where

$$
\begin{aligned}
& \beta_{0}=1.23259, \quad \beta_{2}=2.8978, \\
& \beta_{4}=3.8082, \quad \beta_{22}=0.7150 .
\end{aligned}
$$

The local expansion for $\hat{\psi}_{0}$ through $O\left(\eta^{5}\right)$ and $O\left(\zeta^{5}\right)$ is given below:

$$
\begin{aligned}
\hat{\psi}_{0}(\zeta, \eta)= & {\left[\frac{1}{2 !}\left\{\beta_{0} \zeta+\frac{\beta_{2}}{3 !} \zeta^{3}+\left(\frac{\beta_{4}}{5 !}+\frac{\beta_{22}}{(3 !)^{2}}\right) \zeta^{5}+\cdots\right\}\right] \eta^{2} } \\
& +\left[-\frac{1}{3 !}\left\{\zeta-\frac{1}{3 !} \zeta^{3}+\left(\frac{2}{5 !}+\frac{1}{2(3 !)}\right) \zeta^{5}+\cdots\right] \eta^{3}+O\left(\eta^{5}\right) .\right.
\end{aligned}
$$

In terms of inner variables, (46) becomes

$$
\hat{\psi}_{0}(\zeta, \eta)=\delta^{2} F_{2}(\zeta) \tilde{\eta}^{2}+\delta^{3} F_{3}(\zeta) \tilde{\eta}^{3}+O\left(\delta^{5} \tilde{\eta}^{5}\right)
$$

where $F_{2}(\zeta)$ and $F_{3}(\zeta)$ are the terms in brackets in (46).

The stream function equation in terms of inner variables can now be written by substituting (40) and (47) into equation (39). The result to $O\left(\zeta^{5}\right)$ and $O\left(\eta^{5}\right)$ is given by:

$$
\begin{aligned}
i \frac{\partial \tilde{\chi}}{\partial \tilde{\eta}}-\frac{\partial^{3} \tilde{\chi}}{\partial \tilde{\eta}^{3}}= & -i \operatorname{Cos} \zeta-\delta^{2}\{\operatorname{Cos} 2 \zeta\} \\
& +\delta^{3}\left\{2 F_{2} \frac{\partial \tilde{\chi}}{\partial \zeta}+\tilde{\eta}^{2} F_{2}^{1} \frac{\partial^{2} \tilde{\chi}}{\partial \tilde{\eta}^{2}}-2 \tilde{\eta} F_{2} \frac{\partial^{2} \tilde{\chi}}{\partial \zeta \partial \tilde{\eta}}-2 \tilde{\eta} F_{2}^{\prime} \frac{\partial \tilde{\chi}}{\partial \tilde{\eta}}\right\} \\
& +\delta^{4}\left\{6 \tilde{\eta} F_{3} \frac{\partial \tilde{\chi}}{\partial \zeta}+\tilde{\eta}^{3} F_{3}^{\prime} \frac{\partial^{2} \tilde{\chi}}{\partial \tilde{\eta}^{2}}-3 \tilde{\eta} F_{3} \frac{\partial^{2} \tilde{\chi}}{\partial \zeta \partial \tilde{\eta}}-3 \tilde{\eta}^{2} F_{3}^{\prime} \frac{\partial \tilde{\chi}}{\partial \tilde{\eta}}\right\}+O\left(\delta^{6}\right)
\end{aligned}
$$

The boundary conditions are given by

$$
\frac{\partial \tilde{\chi}}{\partial \tilde{\eta}}=\frac{\partial \tilde{\chi}}{\partial \zeta}=0, \quad \tilde{\eta}=0
$$

and the matching condition is

$$
\lim _{\tilde{\eta} \rightarrow \infty}(\partial \tilde{\chi} / \partial \tilde{\eta})=\lim _{\eta \rightarrow 0}(\partial \hat{\chi} / \partial \eta)
$$


Finally, using (41), we obtain the following set of complementary equations for $\Phi_{n}$ through third order:

$$
\begin{aligned}
& \frac{\partial^{3} \Phi_{0}}{\partial \tilde{\eta}^{3}}-i \frac{\partial \Phi_{0}}{\partial \tilde{\eta}}=i \operatorname{Cos} \zeta, \\
& \frac{\partial^{3} \Phi_{1}}{\partial \tilde{\eta}^{3}}-i \frac{\partial \Phi_{1}}{\partial \tilde{\eta}}=0, \\
& \frac{\partial^{3} \Phi_{2}}{\partial \tilde{\eta}^{3}}-i \frac{\partial \Phi_{2}}{\partial \tilde{\eta}}=\operatorname{Cos} 2 \zeta, \\
& \frac{\partial^{3} \Phi_{3}}{\partial \tilde{\eta}^{3}}-i \frac{\partial \Phi_{3}}{\partial \tilde{\eta}}=2 \eta F_{2} \frac{\partial^{2} \Phi_{0}}{\partial \tilde{\eta} \partial \zeta}+2 \tilde{\eta} F_{2}^{\prime} \frac{\partial \Phi_{0}}{\partial \tilde{\eta}}-2 F_{2} \frac{\partial \Phi_{0}}{\partial \zeta}-\tilde{\eta}^{2} F_{2}^{\prime} \frac{\partial^{2} \Phi_{0}}{\partial \tilde{\eta}^{2}} .
\end{aligned}
$$

The boundary conditions at the surface are simply

$$
\partial \Phi_{n} / \partial \tilde{\eta}=\partial \Phi_{n} / \partial \zeta=0 .
$$

To find the boundary conditions as $\eta \rightarrow \infty$, we must use (50), along with the solutions for the outer region. Noting that $\hat{\psi}_{0, \eta}$ and $\hat{\psi}_{0,5 \eta} \rightarrow 0$ as $\eta \rightarrow 0$ implies the following asymptotic behavior:

$$
\begin{aligned}
& \partial \Phi_{0} / \partial \tilde{\eta} \rightarrow-\operatorname{Cos} \zeta, \quad \partial \Phi_{1} / \partial \tilde{\eta} \rightarrow 0, \\
& \partial \Phi_{2} / \partial \tilde{\eta} \rightarrow i \operatorname{Cos} 2 \zeta, \quad \partial \Phi_{3} / \partial \tilde{\eta} \rightarrow f(\zeta) .
\end{aligned}
$$

The solutions to this system of linear partial differential equations are given below:

$$
\begin{aligned}
\Phi_{0}= & \operatorname{Cos} \zeta\left[-\tilde{\eta}+s^{*}\left(1-e^{-s} \tilde{\eta}\right)\right], \\
\Phi_{1}= & 0, \\
\Phi_{2}= & \operatorname{Cos} 2 \zeta\left[i \tilde{\eta}-s\left(1-e^{-s \eta}\right)\right], \\
\Phi_{3}= & 2 F_{2} \operatorname{Sin} \zeta\left[\frac{9}{4}\left(e^{-s \tilde{\eta}}-1\right)+s \tilde{\eta}+\left(i \frac{\tilde{\eta}^{2}}{4}+s \frac{5}{4} \tilde{\eta}\right) e^{-s \tilde{\eta}}\right] \\
& +F_{2}^{\prime} \operatorname{Cos} \zeta\left[\frac{13}{4}\left(1-e^{-s \tilde{\eta}}\right)-i \tilde{\eta}^{2}+\left(\frac{\tilde{\eta}^{3}}{6 s}-i \frac{5}{4} \tilde{\eta}^{2}-s \frac{13}{4} \tilde{\eta}\right) e^{-s \tilde{\eta}}\right],
\end{aligned}
$$

where $s=(1+i) / \sqrt{2}$ and $s s^{*}=1$. As expected, the first term in the expansion, $\Phi_{0}$, represents a Stokes wave solution in response to the unsteady pressure term and the second term, $\Phi_{2}$, represents a Stokes wave solution to the quasi-steady pressure term.

4.3. Composite solution. To determine the unknown constants in the outer solution, we will apply the matching principle given below:

$$
\lim _{\eta \rightarrow 0} \hat{\chi}^{0}(\zeta, \eta)=\lim _{\tilde{\eta} \rightarrow \infty} \hat{\chi}^{i}(\zeta, \eta) \text { where } \hat{\chi}^{i}=\delta \tilde{\chi}^{i} .
$$

This simply states that the inner expansion of (the outer expansion) equals the outer expansion of (the inner expansion). 
Neglecting exponentially small terms, the inner expansion can be written in outer variables as

$$
\begin{aligned}
\left(\hat{\chi}^{i}\right)^{0} \sim & -\eta \operatorname{Cos} \zeta+\delta\left(s^{*} \operatorname{Cos} \zeta\right)+\delta^{2}\left(i \eta \operatorname{Cos} 2 \zeta-i \eta^{2} F_{2}^{\prime} \operatorname{Cos} \zeta\right) \\
& -\delta^{3}\left(s \operatorname{Cos} 2 \zeta-i \eta^{2} F_{2}^{\prime} \operatorname{Cos} \zeta\right) \\
& +\delta^{4}\left(\frac{13}{4} F_{2}^{\prime} \operatorname{Cos} \zeta-\frac{9}{2} F_{2} \operatorname{Sin} \zeta\right)+O\left(\delta^{5}\right)
\end{aligned}
$$

Similarly, the outer expansion can be expanded as $\eta \rightarrow 0$ as

$$
\begin{aligned}
\left(\hat{\chi}^{0}\right)^{i} \sim & -\eta \operatorname{Cos} \zeta+C_{0}(\zeta)+\delta\left(C_{1}(\zeta)\right) \\
& +\delta^{2}\left(-i \eta^{2} F_{2}^{\prime} \operatorname{Cos} \zeta+i \eta \operatorname{Cos} 2 \zeta+i C_{2}(\zeta)\right) \\
& -\delta^{3}\left(i 2 C_{1}^{\prime}(\zeta) F_{2}-C_{3}(\zeta)\right)+O\left(\delta^{4}\right) .
\end{aligned}
$$

The unknown functions, obtained by matching the two expansions, are given below:

$$
C_{0}=0, \quad C_{1}=s^{*} \operatorname{Cos} \zeta, \quad C_{2}=0, \quad C_{3}=-s \operatorname{Cos} 2 \zeta .
$$

The composite expansion can now be written as

$$
\hat{\chi}^{c}=\hat{\chi}^{i}+\hat{\chi}^{0}-\left(\hat{\chi}^{i}\right)^{0} \text {. }
$$

Using $\left(\hat{\chi}^{i}\right)^{0}$, given by (59), the composite solution can be written as:

$$
\begin{aligned}
\hat{\chi}^{c}= & \delta \Phi_{0}+\delta^{2}\left\{i \operatorname{Sin} \zeta\left(2 \hat{\psi}_{0}-\eta \hat{\psi}_{0, \eta}\right)-i \hat{\psi}_{0, \zeta} \operatorname{Cos} \zeta\right\} \\
+ & \delta^{3}\left\{\Phi_{2}+s \hat{\psi}_{0, \eta} \operatorname{Sin} \zeta\right\}+\delta^{4}\left\{2 F_{2} \operatorname{Sin} \zeta\left(i \frac{\tilde{\eta}^{2}}{4}+s \frac{5}{4} \tilde{\eta}+\frac{9}{4}\right) e^{-s \tilde{\eta}}\right. \\
& \left.+F_{2}^{\prime} \operatorname{Cos} \zeta\left(\frac{\tilde{\eta}^{3}}{6 s}-i \frac{5}{4} \tilde{\eta}^{2}-\frac{13}{4} s \tilde{\eta}+\frac{13}{4}\right) e^{-s \tilde{\eta}}\right\}
\end{aligned}
$$

In (63), the terms neglected are $O\left(\delta^{4}\right)$ in the outer region. However, due to the scaling employed in the inner region, the terms neglected are $O\left(\delta^{5}\right)$. Noting that $\partial / \partial \eta=\delta(\partial / \partial \tilde{\eta})$, and differentiating (63) with respect to $\eta$ yields the time independent, $\zeta$-component of the velocity given by

$$
\begin{aligned}
\frac{\partial \hat{\chi}^{c}}{\partial \eta}= & -\left(1-e^{-s \tilde{\eta}}\right) \operatorname{Cos} \zeta \\
& +i \delta^{2}\left\{\left(1-e^{-s \tilde{\eta}}\right) \operatorname{Cos} 2 \zeta+\left(\hat{\psi}_{0, \eta}-\eta \hat{\psi}_{0, \eta \eta}\right) \operatorname{Sin} \zeta-\hat{\psi}_{0, \zeta \eta} \operatorname{Cos} \zeta\right\} \\
& +\delta^{3}\left\{\left[s \hat{\psi}_{0, \eta \eta}-s\left(1+s \frac{3}{4} \tilde{\eta}-i \frac{\tilde{\eta}^{2}}{4}\right) 2 F_{2} e^{-s \tilde{\eta}}\right] \operatorname{Sin} \zeta\right. \\
& \left.+F_{2}^{\prime} \operatorname{Cos} \zeta\left(i \frac{3}{4} \tilde{\eta}+i s \frac{3}{4} \tilde{\eta}^{2}-\frac{1}{6} \tilde{\eta}^{3}\right) e^{-s \tilde{\eta}}\right\}+O\left(\delta^{4}\right)
\end{aligned}
$$

Notice that (64) satisfies the boundary conditions at the wall and the edge of the boundary layer. This can be shown by noting that $\hat{\psi}_{0, \eta} \rightarrow \operatorname{Sin} \zeta$ and $\hat{\psi}_{0, \zeta \eta} \rightarrow \operatorname{Cos} \zeta$ as $\eta \rightarrow \infty$. Thus, the terms of $O\left(\delta^{2}\right)$ and $O\left(\delta^{3}\right)$ asymptotically go to zero. 
An expression for the nondimensional wall shear stress can also be obtained by differentiating $(64)$ and using $\hat{\psi}_{0, \eta, \eta}(\xi, 0)=2 F_{2}^{\prime}$. The result is

$$
\begin{aligned}
\left.\frac{\partial^{2} \hat{\chi}^{c}}{\partial \eta^{2}}\right|_{\eta=0}= & -\frac{s}{\delta} \operatorname{Cos} \zeta+i s \delta \operatorname{Cos} 2 \zeta \\
& -i \delta\left(\frac{1}{2} F_{2} \operatorname{Sin} \zeta+\frac{5}{4} F_{2}^{\prime} \operatorname{Cos} \zeta\right)+O\left(\delta^{3}\right) .
\end{aligned}
$$

The primary reason for not extending the perturbation solution to higher order is due to the difficulty of obtaining solutions to the stream function in the outer region. Although (29) is a linear first-order equation, the proliferation of the nonhomogeneous terms and the difficulty associated with integrating these terms dictates the order of the perturbation expansion for the stream function. However, two additional terms can readily be added to the perturbation expansion for the $\zeta$-component of velocity and thus the shear stress. This is possible since the $\delta^{4}$ and $\delta^{5}$ velocity expressions are given explicitly by (29). Using the $\phi_{2}$ and $\phi_{3}$ solutions along with (16), the results after simplifying become

$$
\begin{aligned}
\frac{\partial \phi_{4}}{\partial \eta}= & -\left\{3 \hat{U}_{0} \hat{U}_{0 \zeta}+4 \hat{\psi}_{0, \eta \eta \eta}\right\} \operatorname{Sin} \zeta+\left\{2 \hat{\psi}_{0, \eta}-2 \eta \hat{\psi}_{0, \eta \eta}\right\} \operatorname{Sin} 2 \zeta \\
& +\left\{2 \hat{\psi}_{0} \hat{\psi}_{0, \eta \eta}-\hat{\psi}_{0, \eta}^{2}+\left(\hat{U}_{0} \hat{U}_{0 \zeta}\right)_{\zeta}\right\} \operatorname{Cos} \zeta-\hat{\psi}_{0, \zeta \eta} \operatorname{Cos} 2 \zeta
\end{aligned}
$$

and

$$
\frac{\partial \phi_{5}}{\partial \eta}=-i C_{3}^{\prime}(\zeta) \hat{\psi}_{0, \eta \eta} .
$$

The corresponding solution in the inner region is easily found by solving the following two equations:

$$
\begin{gathered}
\frac{\partial^{3} \Phi_{4}}{\partial \tilde{\eta}^{3}}-i \frac{\partial \Phi_{4}}{\partial \tilde{\eta}}=3 \tilde{\eta}^{2} F_{3} \frac{\partial^{2} \Phi_{0}}{\partial \zeta \partial \tilde{\eta}}+3 \tilde{\eta}^{2} F_{3}^{\prime} \frac{\partial \Phi_{0}}{\partial \tilde{\eta}}-6 \tilde{\eta} F_{3} \frac{\partial \Phi_{0}}{\partial \zeta}-\tilde{\eta}^{3} F_{3}^{\prime} \frac{\partial^{2} \Phi_{0}}{\partial \tilde{\eta}^{2}} \\
\frac{\partial^{3} \Phi_{5}}{\partial \tilde{\eta}^{3}}-i \frac{\partial \Phi_{5}}{\partial \tilde{\eta}}=2 \tilde{\eta} F_{2} \frac{\partial^{2} \Phi_{2}}{\partial \zeta \partial \tilde{\eta}}+2 \tilde{\eta} F_{2}^{\prime} \frac{\partial \Phi_{2}}{\partial \tilde{\eta}}-2 F_{2} \frac{\partial \Phi_{2}}{\partial \zeta}-\tilde{\eta}^{2} F_{2}^{\prime} \frac{\partial^{2} \Phi_{2}}{\partial \tilde{\eta}^{2}}
\end{gathered}
$$

Requiring that $\partial \Phi_{n} / \delta \tilde{\eta}$ be zero at the surface and finite as $\tilde{\eta} \rightarrow \infty$ yields the following two solutions:

$$
\begin{aligned}
\frac{\partial \Phi_{4}}{\partial \tilde{\eta}}= & 3 F_{3} \operatorname{Sin} \zeta\left[2\left(e^{-s \tilde{\eta}}-1\right)+2 s \tilde{\eta}-i \tilde{\eta}^{2}-s^{*}\left(i \frac{3}{4} \tilde{\eta}+i s \frac{3}{4} \tilde{\eta}^{2}-\frac{1}{6} \tilde{\eta}^{3}\right) e^{-s \tilde{\eta}}\right] \\
& +3 F_{3}^{\prime} \operatorname{Cos} \zeta\left[-i \tilde{\eta}^{2}-2\left(1-e^{-s \tilde{\eta}}\right)+\left(s \frac{3}{8} \tilde{\eta}+i \frac{3}{8} \tilde{\eta}^{2}-\frac{\tilde{\eta}^{3}}{4 s}-\frac{\tilde{\eta}^{4}}{24}\right) e^{-s \tilde{\eta}}\right], \\
\frac{\partial \Phi_{5}}{\partial \tilde{\eta}}= & -i 4 F_{2} \operatorname{Sin} \zeta\left[s-s\left(1+s \frac{3}{4} \tilde{\eta}+i \frac{\tilde{\eta}^{2}}{4}\right) e^{-s \tilde{\eta}}\right] \\
& -i F_{2}^{\prime} \operatorname{Cos} \zeta\left[-i 2 \tilde{\eta}+\left(i \frac{3}{4} \tilde{\eta}+i s \frac{3}{4} \tilde{\eta}^{2}-\frac{\tilde{\eta}^{3}}{6}\right) e^{-s \tilde{\eta}}\right] .
\end{aligned}
$$


The composite velocity is then determined by using these results along with the previous solution through $O\left(\delta^{3}\right)$ and applying the matching principle given by

$$
\lim _{\eta \rightarrow 0}\left(\partial \hat{\chi}^{0} / \partial \eta\right)=\lim _{\tilde{\eta} \rightarrow \infty}\left(\partial \hat{\chi}^{i} / \partial \eta\right) .
$$

Neglecting the exponentially small terms and using both (16) and (47),

$$
\begin{aligned}
\left(\frac{\partial \hat{\chi}^{i}}{\partial \eta}\right)^{0}= & \left(\frac{\partial \hat{\chi}^{0}}{\partial \eta}\right)^{i} \sim-\operatorname{Cos} \zeta+i \delta^{2}\left\{\operatorname{Cos} 2 \zeta-2 F_{2}^{\prime} \eta \operatorname{Cos} \zeta-3 \eta^{2} F_{3} \operatorname{Sin} \zeta\right. \\
& \left.-3 \eta^{2} F_{3}^{\prime} \operatorname{Cos} \zeta\right\}+s \delta^{3}\left\{2 F_{2} \operatorname{Sin} \zeta+6 F_{3} \eta \operatorname{Sin} \zeta\right\} \\
& -\delta^{4}\left\{6 F_{3} \operatorname{Sin} \zeta+6 F_{3}^{\prime} \operatorname{Cos} \zeta+2 \eta F_{2}^{\prime} \operatorname{Cos} 2 \zeta\right\} \\
& +s^{*} \delta^{5}\left\{4 F_{2} \operatorname{Sin} 2 \zeta\right\}+O\left(\delta^{6}\right) .
\end{aligned}
$$

The composite velocity can then be written as

$$
\partial \hat{\chi}^{c} / \partial \eta=\partial \hat{\chi}^{0} / \partial \eta+\partial \hat{\chi}^{i} / \partial \eta-\left(\partial \hat{\chi}^{i} / \partial \eta\right)^{0} .
$$

Using (72) and introducing the new variable $\bar{\eta} \equiv s \eta$ and $\bar{\delta} \equiv \delta / s$, the composite velocity expansion becomes

$$
\begin{aligned}
\frac{\partial \hat{\chi}^{c}}{\partial \eta}= & -\left(1-e^{-\bar{\eta}}\right) \operatorname{Cos} \zeta \\
& -\bar{\delta}^{2}\left\{\left(1-e^{-\bar{\eta}}\right) \operatorname{Cos} 2 \zeta-\hat{\psi}_{0, \zeta \eta} \operatorname{Cos} \zeta+\left(\hat{\psi}_{0, \eta}-\eta \hat{\psi}_{0, \eta \eta}\right) \operatorname{Sin} \zeta\right\} \\
& -\bar{\delta}^{3}\left\{\left(\hat{\psi}_{0, \eta \eta}-2 F_{2} P_{1}\right) \operatorname{Sin} \zeta+F_{2}^{\prime} P_{2} \operatorname{Cos} \zeta\right\} \\
& -\bar{\delta}^{4}\left\{\phi_{4, \eta}+3 F_{3}\left(2 e^{-\bar{\eta}}-P_{2}\right) \operatorname{Sin} \zeta+3 F_{3}^{\prime}\left(2 e^{-\bar{\eta}}+P_{3}\right) \operatorname{Cos} \zeta\right\} \\
& +\bar{\delta}^{5}\left\{\left(2 \hat{\psi}_{0, \eta \eta}-4 F_{2} P_{1}\right) \operatorname{Sin} 2 \zeta-F_{2}^{\prime} P_{2} \operatorname{Cos} 2 \zeta\right\}+O\left(\bar{\delta}^{6}\right),
\end{aligned}
$$

where

$$
\begin{aligned}
& P_{1}=\left(1+\frac{3}{4} \bar{\eta}+\frac{\bar{\eta}^{2}}{4}\right) e^{-\bar{\eta}} \\
& P_{2}=\left(\frac{3}{4} \bar{\eta}+\frac{3}{4} \bar{\eta}^{2}+\frac{\bar{\eta}^{3}}{6}\right) e^{-\bar{\eta}} \\
& P_{3}=\left(\frac{3}{8} \bar{\eta}+\frac{3}{8} \bar{\eta}^{2}+\frac{\bar{\eta}^{3}}{4}+\frac{\bar{\eta}^{4}}{24}\right) e^{-\bar{\eta}}
\end{aligned}
$$

Finally, the nondimensional wall shear stress can be written through $O\left(\bar{\delta}^{4}\right)$ as

$$
\begin{aligned}
\left.\frac{\partial^{2} \hat{\chi}^{c}}{\partial \eta^{2}}\right|_{\eta=0}= & -\frac{\operatorname{Cos} \zeta}{\bar{\delta}}-\bar{\delta} \operatorname{Cos} 2 \zeta+\bar{\delta}^{2}\left(\frac{5}{4} F_{2}^{\prime} \operatorname{Cos} \zeta-\frac{1}{2} F_{2} \operatorname{Sin} \zeta\right) \\
& +\bar{\delta}^{3}\left(\frac{3}{8} F_{3} \operatorname{Sin} \zeta+\frac{39}{8} F_{3}^{\prime} \operatorname{Cos} \zeta\right)+\bar{\delta}^{4}\left(\frac{5}{4} F_{2}^{\prime} \operatorname{Cos} 2 \zeta+F_{2} \operatorname{Sin} 2 \zeta\right)
\end{aligned}
$$


The nondimensional shear stress and typical velocity distributions are given in Sec. 5 along with a comparison to the numerical solution. However, before presenting these results, it is useful to compare these solutions to Glauert's classical result valid as $\zeta \rightarrow 0$.

4. Stagnation point solutions. The high-frequency solution given in the previous section is valid to $O\left(\zeta^{7}\right)$ in a region about the mean stagnation point. If we now consider the case of an oscillating flow approaching a flat plate, the solution given by (73) can be compared to the well-known solution given by Glauert [7]. Under these conditions, the free stream velocity is given by

$$
\hat{U}_{e}(\zeta, \tau)=\zeta-e^{i \tau}
$$

Alternatively, this can be taken to represent the case of a transversely oscillating free stream approaching a fixed cylinder where terms of $O\left(\zeta^{3}\right)$ and $O\left(\varepsilon \zeta^{2}\right)$ have been neglected. Comparing (75) with (14), we see that $\hat{U}_{0}(\zeta)=\zeta$ and $\hat{U}_{1}(\tau)=-e^{i \tau}$.

Under these conditions, terms involving Sin $\zeta$ in (73) vanish, $\chi_{0}(\zeta, \eta) \rightarrow \zeta f_{0}(\eta)$, and $\chi(\zeta, \eta) \rightarrow \chi(\eta)$. Equation (73) then becomes

$$
\begin{aligned}
\frac{d \hat{\chi}^{c}}{d \eta}= & -\left(1-e^{-\bar{\eta}}\right)-\bar{\delta}^{2}\left(1-e^{-\bar{\eta}}-f_{0}^{\prime}\right) \\
& -\bar{\delta}^{3}\left(\frac{3}{8} \bar{\eta}+\frac{3}{8} \bar{\eta}^{2}+\frac{1}{12} \bar{\eta}^{3}\right) e^{-\bar{\eta}} \\
& -\bar{\delta}^{4}\left(1-f_{0}^{\prime}-e^{-\bar{\eta}}-\left(\frac{3}{16} \bar{\eta}+\frac{3}{8} \bar{\eta}^{2}+\frac{\bar{\eta}^{3}}{8}+\frac{\bar{\eta}^{4}}{48}\right) e^{-\bar{\eta}}\right) \\
& -\bar{\delta}^{5}\left(\frac{3}{8} \bar{\eta}+\frac{3}{8} \bar{\eta}^{2}+\frac{\bar{\eta}^{2}}{12}\right) e^{-\bar{\eta}}+O\left(\bar{\delta}^{6}\right) .
\end{aligned}
$$

In obtaining (76), the following results are used:

$$
\begin{aligned}
\phi_{4, \eta} & \rightarrow 1-f_{0}^{\prime}, \\
F_{2}^{\prime}(\zeta) & \rightarrow \beta_{0} / 2,
\end{aligned}
$$

and

$$
F_{3}^{\prime}(\zeta) \rightarrow-1 / 6
$$

In order to show that this result agrees with the flat plate solution, the essential details of Glauert's analysis will be outlined in terms of the nondimensional variables employed in this analysis. Letting the subscript, $G$, denote variables in Glauert's analysis yields the following relations.

Expanding the stream function as

$$
\hat{\psi}(\zeta, \eta, \tau)=\zeta f_{0}(\eta)-\varepsilon \chi_{G}(\eta) e^{i \tau}
$$

and substituting (77) into (10) yields

$$
f_{0}^{\prime \prime \prime}+f_{0} f_{0}^{\prime \prime}-f_{0}^{\prime 2}=1
$$

and

$$
\chi_{G}^{\prime \prime \prime}+f_{0} \chi_{G}^{\prime \prime}-f_{0} \chi_{G}^{\prime}-i \alpha \chi_{G}^{\prime}=-i \alpha-1
$$


The boundary conditions for (79) are

$$
\chi_{G}(0)=\chi_{G}^{\prime}(0)=0 \quad \text { and } \quad \chi_{G}^{\prime}(\eta \rightarrow \infty) \rightarrow 1 .
$$

To be consistent with Glauert's solution, the following transformation is introduced:

$$
\chi_{G}^{\prime}=\frac{f_{0}^{\prime}-i \alpha_{G}+i \alpha_{G} \phi_{G}}{1-i \alpha_{G}} .
$$

Substituting this into (79) and (80) yields

$$
\begin{gathered}
\phi_{G}^{\prime \prime}+f_{0} \phi_{G}^{\prime}-f_{0} \phi_{G}-i \alpha \phi_{G}=0, \\
\phi_{G}(0)=1, \text { and } \phi_{G}(\eta \rightarrow \infty) \rightarrow 1 .
\end{gathered}
$$

The high-frequency solution is then obtained by expanding $\phi_{G}$ in terms of $\alpha_{G}$ as

$$
\phi_{G}=\sum_{n=0}^{N} \alpha_{G}^{n} \varphi_{n}\left(Y_{G}\right)+O\left(\alpha_{G}^{N+1}\right) \text {. }
$$

The result is given by

$$
\begin{aligned}
\chi_{G}^{\prime}\left(Y_{G}\right)= & \left(1-\varphi_{0}\right)+\alpha_{G}^{2}\left(1-\varphi_{0}-f_{0}^{\prime}\right)+\alpha_{G}^{3}\left(-\varphi_{3}\right) \\
& +\alpha_{G}^{4}\left(1-f_{0}^{\prime}-\varphi_{0}-\varphi_{4}\right)+\alpha_{G}^{5}\left(-\varphi_{3}\right)+O\left(\alpha_{G}^{6}\right),
\end{aligned}
$$

where

$$
\begin{aligned}
\varphi_{0} & =e^{-Y_{G}}, \\
\varphi_{3} & =-\beta_{0}\left(\frac{3}{8} Y_{G}+\frac{3}{8} Y_{G}^{2}+\frac{1}{12} Y_{G}^{3}\right) e^{-Y_{G}},
\end{aligned}
$$

and

$$
\varphi_{4}=\left(\frac{3}{16} Y_{G}+\frac{3}{16} Y_{G}^{2}+\frac{1}{8} Y_{G}^{3}+\frac{1}{48} Y_{G}^{4}\right) e^{-Y_{G}} .
$$

Comparing (76) with (85) we find that the results agree exactly as expected. The difference in sign is due to the minus sign in (77).

It can also be shown by taking the limit as $\zeta \rightarrow 0$ that the unsteady wall shear stress given by (65) agrees exactly with the results given by Glauert [7].

Several observations should be made regarding the special case when curvature is absent. First, $\varepsilon$ becomes arbitrary, since the unsteady solution is no longer a function of the $\zeta$-coordinate, and the unsteady component represents an "exact" solution. Thus, there are no higher or lower time harmonics present as would occur in the present analysis if the expansion in $\varepsilon$ were continued to second order.

Another interesting feature is that the high-frequency expansion for the flat plate can be obtained without the use of an inner and outer expansion. Although the high-frequency expansion is singular, a simple (Stokes layer) coordinate stretching given by $Y_{G}=\eta / \alpha_{G}$ is sufficient to remove the singularity. This fundamental difference between the two problems can be explained from the nonhomogeneous terms in (17) or the transformed version given by (39). For the cylinder, the quasi-steady pressure gradient produces spatial harmonics, i.e., $\operatorname{Cos} 2 \zeta$, which are explicitly present in equation (48). In addition, the convective acceleration terms, which represent the interaction between the mean steady flow and the unsteady flow, produce additional harmonics. These appear as the Sin $\zeta$ 
terms in (58). This interaction continues; for example, the $\phi_{4}$ solution contains $\operatorname{Cos} \zeta \operatorname{Cos} 2 \zeta$ interaction terms. It is impossible for this spatial variation to satisfy the boundary condition at the edge of the boundary layer. Consequently, from a mathematical perspective, an inner region must be defined to accommodate this behavior. This is consistent with the physics of the problem, since the oscillations are known to exist only in a thin layer adjacent to the cylinder for high reduced frequencies.

Applying higher-order boundary layer theory to account for the displacement thickness does not alter these differences. Since the displacement thickness is constant for the infinite flat plate, there is no mechanism present to produce a spatial variation in the unsteady pressure gradient.

From these observations, it can be concluded that there is a fundamental difference between these two geometries in the response of the boundary layer to oscillations.

The similarity of the unsteady velocity and shear stress holds only at the stagnation point. Differences between the two flows occur quite rapidly along the surface of the cylinder. Consequently, interpreting unsteady effects in the neighborhood of the cylinder by extrapolating results from the flat plate is not reasonable as Glauert [7] among others has suggested.

5. Results and discussion. The unsteady response of a laminar incompressible boundary layer described by (17) and (19) has been solved by the method of matched asymptotic expansions. The time-independent component of the stream function given by (63) is valid for high reduced frequencies to $O\left(\delta^{5}\right)$. In addition, it is formally accurate to $O\left(\zeta^{7}\right)$ in a region about the mean stagnation point. The time-independent composite expansions for the velocity and wall shear stress given by (73) and (74) are valid to $O\left(\delta^{6}\right)$ and $O\left(\delta^{5}\right)$, respectively. These solutions have been shown to agree exactly with Glauert's planar stagnation point solution as $\delta \rightarrow 0$. In order to validate the asymptotic solution for nonzero values of $\zeta$, it is necessary to solve (16) and (17) numerically.

The nonsimilar boundary-layer equation given by (16) can be solved by a variety of methods as discussed by Cebeci and Bradshaw [3]. However, to be consistent with the perturbation analysis, (16) was solved by the Howarth type expansion. In addition, this classical approach yields very accurate solutions for bluff bodies with minimal computational effort in a region about the mean stagnation point where comparisons with the asymptotic expansion are desired.

When solving the disturbance equation given by (17) it is important that the numerical algorithm be able to accurately resolve the fine scale structure near the wall. This is especially true when computing values of the wall shear stress. For this reason, special attention was given to the numerical solution of this equation. The equation was solved for arbitrary frequencies by two different methods.

The techniques were sufficiently unique to warrant discussion, and the details are outlined in the Appendix. The only similarity was that both methods involved variable computational points in the $\eta$-direction which were determined by a specified error tolerance. The numerical results presented in this paper are based upon the solution of equations (A.17)-(A.20) which result from the Howarth type expansion. The convergence of this solution was carefully tested by comparing the three-term expansion, i.e., $\phi_{0}, \phi_{2}$, and $\phi_{4}$ equations, with the four-term expansion which includes the $\phi_{6}$ equation. The 
convergence criteria was based upon the relative error in the wall shear stress between the three- and four-term expansion. Calculations were made for $0 \leqslant \alpha \leqslant 50$ and $\delta \leqslant 1.0$ As expected, the maximum relative error occured at $\zeta=1.0$. The error was a weak function of $\alpha$ and varied from $1.3 \%$ at $\alpha=1$ to less than $1.0 \%$ for $\alpha \geqslant 10.0$. Based upon these results and calculations using the semi-discrete method, the numerical solution can be taken as a benchmark for comparisons with the perturbation solution.

The results, which show the most interesting features of the unsteady flow, are velocity profiles and wall shear stress. Because of the exponential time factor, $e^{i \tau}$, used in obtaining the asymptotic and numerical solutions, these results are given by the real and imaginary parts of $\chi_{\eta}(\zeta, \eta)$ and $\chi_{\eta \eta}(\zeta, 0)$. Due to the physical limitations inherent in the model, results are given only for $\zeta \leqslant 1$.
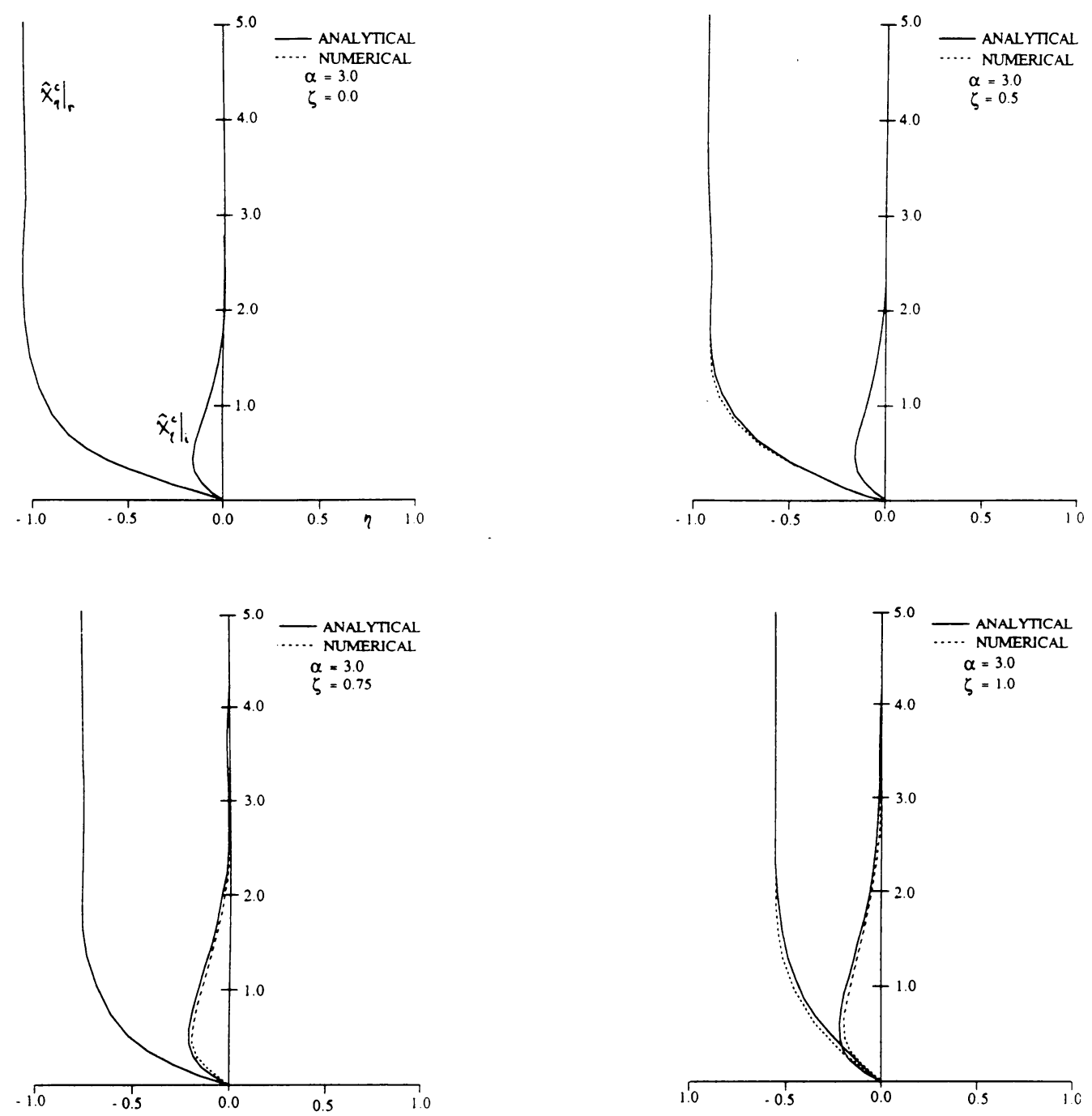

FIG. 3. Comparison of real and imaginary parts of $\hat{\chi}_{\eta}^{c}$ vs. $\eta$. 

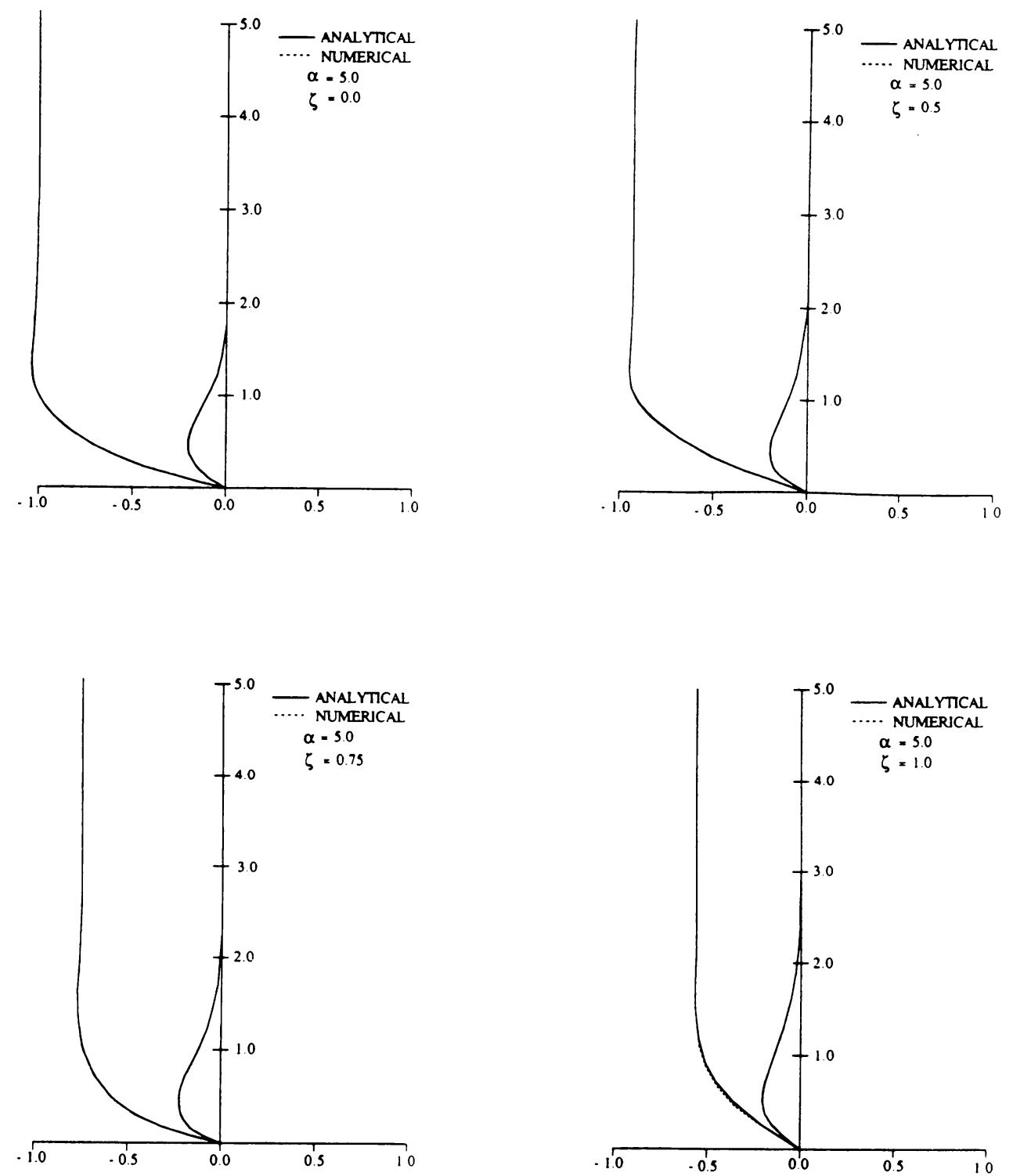

FIG. 4. Comparison of real and imaginary parts of $\hat{\chi}_{\eta}^{c}$ vs. $\eta$. 

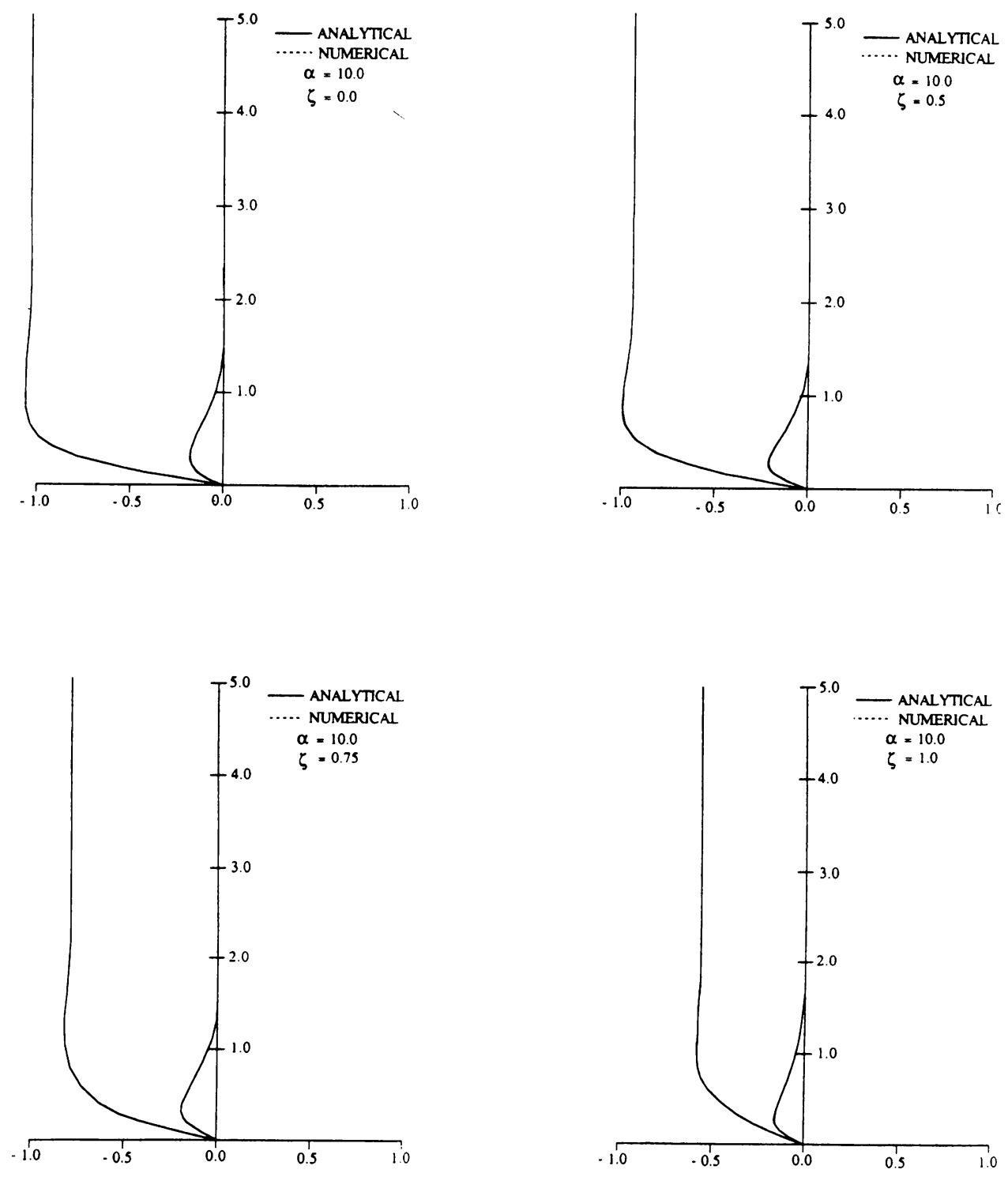

FIG. 5. Comparison of real and imaginary parts of $\hat{\chi}_{\eta}^{c}$ vs. $\eta$. 
Typical "velocity" profiles shown in Figs. 3 through 5 represent the real and imaginary parts of equation (64) for various reduced frequencies. For values of $\alpha \geqslant 3$, there is no discernible difference between the numerical and perturbation solution provided $\zeta \leqslant 0.5$, where for $\alpha \geqslant 5$, the two solutions agree up to $\zeta \leqslant 1.0$ within the resolution provided by the plots. This $\zeta$ dependency, which is more clearly seen in the wall shear stress, was unexpected. However, the reason for this behavior becomes apparent by examining the perturbation solution given by (73). In obtaining this composite expansion, the steady mean flow was approximated by a local expansion in both $\tilde{\eta}$ and $\zeta$ given by (47). This expansion was used in the inner region to represent the coefficients in (39). As $\zeta \rightarrow 0$, the contributions from these steady-unsteady convective interaction terms become increasingly less important. In fact, at $\zeta=0$, only two interaction terms remain, as seen in (76). Now, as $\alpha \rightarrow 1$, the thickness of the unsteady inner region becomes comparable to the steady boundary layer. Not only does the perturbation expansion in $\alpha$ break down, but also the local expansion for the steady flow in $\tilde{\eta}$ begins to fail. This effect becomes more pronounced as $\zeta \rightarrow 1.0$, hence the reason for the small departures in the velocity profiles shown in Fig. 3. This breakdown is even more apparent for $\alpha=2$ as shown in Fig. 6 for $\zeta=.25$ and $\zeta=1.0$. However, when $\alpha \geqslant 5$, the agreement for $0 \leqslant \zeta \leqslant 1$ is excellent, as demonstrated by both velocity profiles and wall shear stress comparisons.

It should be noted that the velocity profiles shown in Figs. 3 through 6 are based upon a four-term expansion, although the solution given by (73) contains five terms. The reason for this is that even-term expansions, i.e., two and four, vs. odd-term, i.e., three and five, generally provided better agreement with the numerical solution. An explanation for this behavior can be offered by investigating each individual term in the perturbation expansion. In the inner solution, each consecutive term represents a viscous wave which responds to a particular harmonic, i.e., $\operatorname{Cos} \zeta, \operatorname{Cos} 2 \zeta$, etc., in the $\zeta$-direction. In addition, each wave has its own unique phase shift in the $\eta$-direction due to the complex form of $P_{1}$,
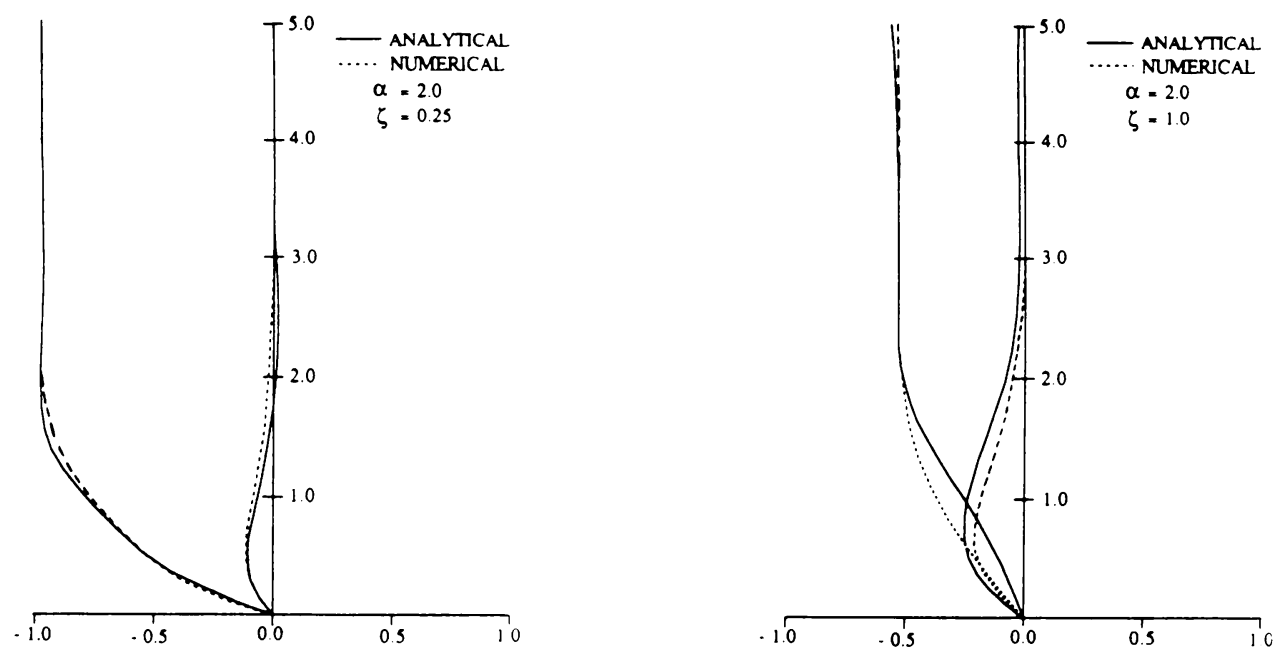

Fig. 6. Comparison of real and imaginary parts of $\hat{\chi}_{\eta}^{c}$ vs. $\eta$. 

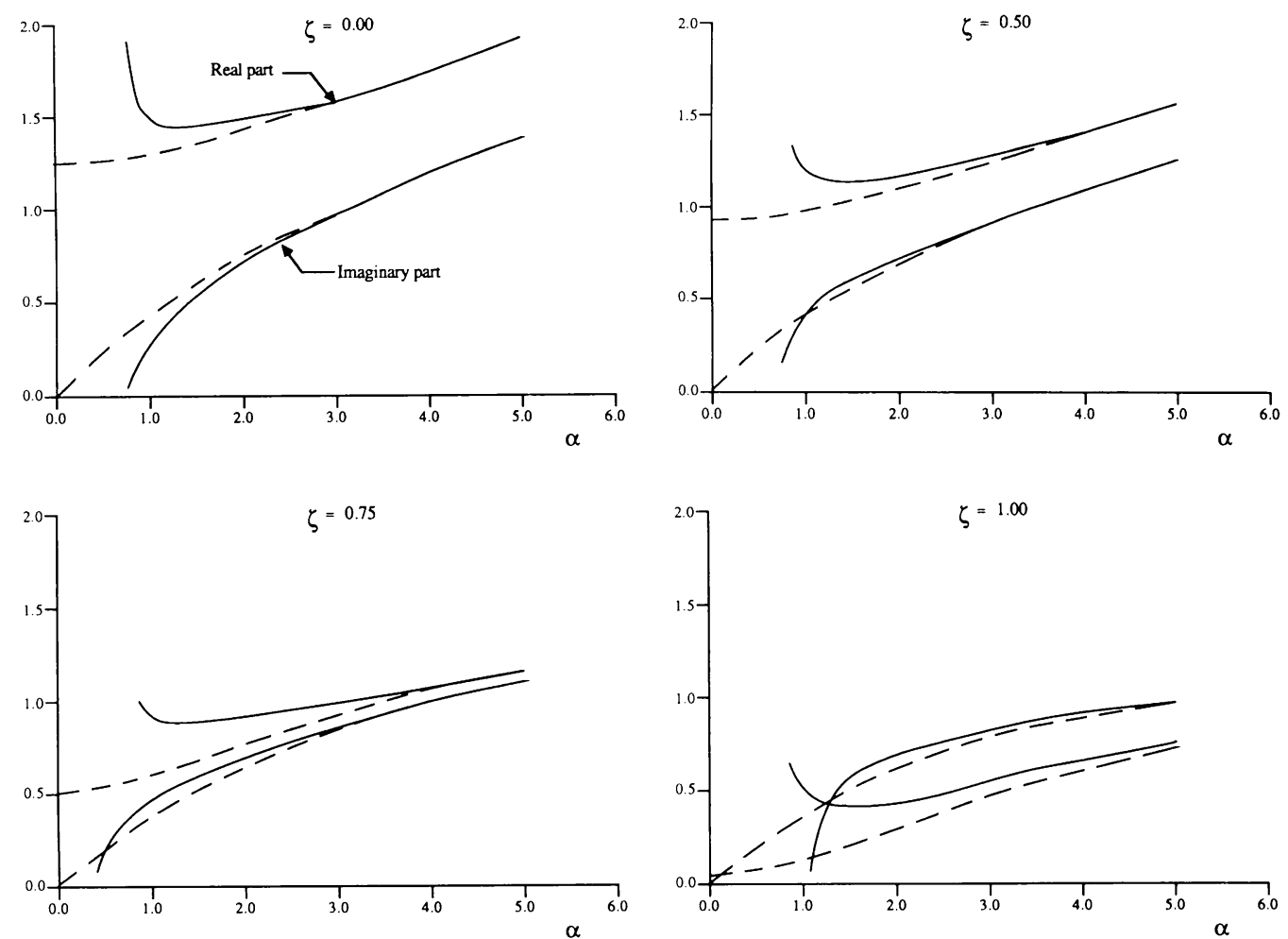

FIG. 7. Comparison of real and imaginary parts of $\hat{\chi}_{\eta \eta}^{c}(0, \zeta)$ vs. $\alpha$. (-Analytical, --Numerical)

$P_{2}, P_{3}$. The overall effect is quite complicated; however, a numerical investigation of each term in the perturbation expansion provided some insight into the manifestation of this phenomenon. The numerical study indicated that the general trend at a constant $\eta$ for each consecutive term is one of alternating sign. This suggests that an expansion which contains an even number of harmonics is preferable. This a posteriori explanation is not conclusive; in fact, on occasion, the five-term expansion gave slightly better agreement than the four-term expansion. Nevertheless, the results for the perturbation expansion shown in Figs. 3 through 7 are based upon the four-term expansion exclusively.

Fig. 7 shows the real and imaginary values of $\tilde{\chi}_{\eta \eta}^{c}(\zeta, 0)$ vs. $\alpha$ for various values of $\zeta$. In general, both components have a monotonic increase in magnitude with increasing $\alpha$. The variation of the phase advance of the shear stress relative to the velocity fluctuation is also seen to steadily increase from 0 to $\pi / 4$ as $\alpha$ increases. This effect is a strong function of position and is most pronounced at $\zeta=\pi / 4$. At this location, the phase advance is almost independent of $\alpha$ for $\alpha>1$. It is interesting that this effect can be predicted by considering only the first two terms in (65). Using $s=(1+i) / \sqrt{ } 2$, the nondimensional shear stress becomes

$$
\left.\frac{\partial^{2} \chi}{\partial \eta^{2}}\right|_{\eta=0}=\frac{1}{\sqrt{2}}\left\{\left(\frac{\operatorname{Cos} \zeta}{\delta}-\delta \operatorname{Cos} 2 \zeta\right)+i\left(\frac{\operatorname{Cos} \zeta}{\delta}+\delta \operatorname{Cos} 2 \zeta\right)\right\}+O\left(\delta^{2}\right) .
$$


Setting $\zeta=\pi / 4$, we find that the phase advance is exactly $\pi / 4$ to $O\left(\delta^{2}\right)$ which agrees quite well with the numerical solution.

In conclusion, perturbation analysis, and in particular the method of matched asymptotic expansions, has provided a useful technique for investigating the unsteady behavior of boundary layers. It not only resolves the fine scale structure near the wall but provides valuable insight into the physical mechanisms responsible for this structure. In addition, the formulation provides the flexibility to investigate not only vertical but also arbitrary translations of the cylinder. For this case, the inviscid stream function given by (6) would be used to determine a new edge velocity. The subsequent perturbation analysis for the unsteady boundary layer is altered only by the boundary condition and the nonhomogeneous pressure gradient term. Rotational oscillations could be superimposed to obtain yet another inviscid stream function. This latter case could be useful in determining the wall shear stress and subsequent heat transfer coefficients for whirling oscillations encountered by cylindrical tube heat exchangers subjected to cross flow.

Appendix. Numerical solution for arbitrary frequencies. In order to verify the matched asymptotic expansion, a numerical solution to equations (16) and (17) was obtained. To be consistent with the perturbation analysis, (16) was solved by an expansion in terms of the $\zeta$-coordinate. Equation (17) was solved by a similar type expansion and also by a semi-discrete method. The notation and the coefficients employed in solving (16) differ from those usually given for the Blasius series. Consequently, the details for this solution are outlined along with those for the unsteady solution.

The form of the pressure gradient given in (16) suggests the following expansion for $\hat{\psi}_{0}(\zeta, \eta)$ :

$$
\begin{aligned}
\hat{\psi}_{0}(\zeta, \eta)= & f_{0}(\eta) \zeta+a_{2} f_{2}(\eta) \zeta^{3}+\left[a_{4} f_{4}(\eta)+a_{2}^{2} f_{22}(\eta)\right] \zeta^{5} \\
& +\left[a_{6} f_{6}(\eta)+a_{2} a_{4} f_{42}(\eta)+a_{2}^{3} f_{222}(\eta)\right] \zeta^{7}+\cdots .
\end{aligned}
$$

The constants in this equation are obtained by matching the edge boundary condition, i.e., $\partial \hat{\psi}_{0} / \partial \eta \rightarrow \hat{U}_{0}(\zeta)$ as $\eta \rightarrow \infty$, and requiring that $f_{n}^{\prime}(\eta \rightarrow \infty) \rightarrow 1$ while the remaining functions of $\eta$ must vanish. This yields the following relations for the constants:

$$
a_{2}=-1 / 3 \text { ! }, a_{4}=1 / 5 \text { !, } a_{6}=-1 / 7 \text { !, etc. }
$$

Finally, substituting (A.1) into (16) and collecting equal powers of $\zeta$ yields the following coupled boundary value problems:

where

$$
\begin{aligned}
& f_{0}^{\prime \prime \prime}+f_{0} f_{0}^{\prime \prime}-f_{0}^{\prime 2}=-1, \\
& f_{2}^{\prime \prime \prime}+f_{0} f_{2}^{\prime \prime}-4 f_{0}^{\prime} f_{2}^{\prime}+3 f_{0}^{\prime \prime} f_{2}=-4, \\
& f_{4}^{\prime \prime \prime}+f_{0} f_{4}^{\prime \prime}-6 f_{0}^{\prime} f_{4}^{\prime}+5 f_{0}^{\prime \prime} f_{4}=-6, \\
& f_{6}^{\prime \prime \prime}+f_{0} f_{6}^{\prime \prime}-8 f_{0}^{\prime} f_{6}^{\prime}+7 f_{0}^{\prime \prime} f_{6}=-8,
\end{aligned}
$$

$$
f_{42}^{\prime \prime \prime}+f_{0} f_{42}^{\prime \prime}-8 f_{0}^{\prime} f_{42}^{\prime}+7 f_{0}^{\prime \prime} f_{42}=-8+\left(8 f_{2}^{\prime} f_{4}^{\prime}-5 f_{2}^{\prime \prime} f_{4}-3 f_{2} f_{4}^{\prime \prime}\right) \text {, }
$$




$$
f_{222}^{\prime \prime \prime}+f_{0} f_{222}^{\prime \prime}-8 f_{0}^{\prime} f_{222}^{\prime}+7 f_{0}^{\prime \prime} f_{222}=\left(8 f_{2}^{\prime} f_{22}^{\prime}-5 f_{2}^{\prime \prime} f_{22}-3 f_{2} f_{22}^{\prime \prime}\right),
$$

where

$$
f(0)=f^{\prime}(0) \text { and } \quad f^{\prime}(\eta \rightarrow \infty) \rightarrow 0 .
$$

The same methodology can be applied to (17), after first separating the time behavior by writing $\hat{\psi}_{1}(\zeta, \eta, \tau)=\hat{\chi}(\zeta, \eta) e^{i \tau}$. The unsteady disturbance equation can then be written as

$$
L_{2} \frac{\partial \hat{\chi}}{\partial \eta}+L_{1} \frac{\partial \hat{\chi}}{\partial \zeta}=-i \alpha \operatorname{Cos} \zeta-\operatorname{Cos} 2 \zeta
$$

where

$$
L_{2} \equiv i \alpha+\hat{\psi}_{0, \zeta \eta}-\hat{\psi}_{0,5} \frac{\partial}{\partial \eta}-\frac{\partial^{2}}{\partial \eta^{2}}
$$

and

$$
L_{1} \equiv \hat{\psi}_{0, \eta} \frac{\partial}{\partial \eta}-\hat{\psi}_{0, \eta \eta}
$$

The nonhomogeneous term suggests the following expansion for $\hat{\chi}(\zeta, \eta)$ :

$$
\hat{\chi}(\zeta, \eta)=b_{0} \phi_{0}(\eta)+b_{2} \phi_{2}(\eta) \zeta^{2}+b_{4} \phi_{4}(\eta) \zeta^{4}+b_{6} \phi_{6}(\eta) \zeta^{6}+\cdots .
$$

As before, the constants are determined by matching the edge boundary condition. Using $\partial \hat{\chi} / \partial \eta \rightarrow-\operatorname{Cos} \zeta$, for the case of an oscillating cylinder, yields the following relations:

$$
b_{0}=-1, b_{2}=1 / 2 !, b_{4}=1 / 4 !, b_{6}=1 / 6 ! \cdots .
$$

Finally, using the expansion given by (A.15) and expanding the nonhomogeneous term in a Taylor series yields the following boundary value problems:

$$
\begin{gathered}
\phi_{0}^{\prime \prime \prime}+f_{0} \phi_{0}^{\prime \prime}-f_{0}^{\prime} \phi_{0}^{\prime}-i \alpha \phi_{0}^{\prime}=-1-i \alpha, \\
\phi_{2}^{\prime \prime \prime}+f_{0} \phi_{2}^{\prime \prime}-3 f_{0}^{\prime} \phi_{2}^{\prime}-i \alpha \phi_{2}^{\prime}+2 f_{0}^{\prime \prime} \phi_{2}=-4-\imath \alpha+f_{2}^{\prime} \phi_{0}^{\prime}-f_{2} \phi_{0}^{\prime \prime} \\
\phi_{4}^{\prime \prime \prime}+f_{0} \phi_{4}^{\prime \prime}-5 f_{0}^{\prime} \phi_{4}^{\prime}-i \alpha \phi_{4}^{\prime}+4 f_{0}^{\prime \prime} \phi_{4}=-16-\imath \alpha \\
+\left(f_{4}^{\prime}+\frac{10}{3} f_{22}^{\prime}\right) \phi_{0}^{\prime}-\left(f_{4}+\frac{10}{3} f_{22}\right) \phi_{0}^{\prime \prime}-4 f_{2}^{\prime \prime} \phi_{2}+10 f_{2}^{\prime} \phi_{2}^{\prime}-6 f_{2} \phi_{2}^{\prime \prime} \\
\phi_{6}^{\prime \prime \prime}+f_{0} \phi_{6}^{\prime \prime}-7 f_{0}^{\prime} \phi_{6}^{\prime}-i \alpha \phi_{6}^{\prime}+6 f_{0}^{\prime \prime} \phi_{6}=-64-\imath \alpha \\
+\left(f_{6}^{\prime}+7 f_{42}^{\prime}+\frac{70}{3} f_{222}^{\prime}\right) \phi_{0}^{\prime}-\left(f_{6}+7 f_{42}+\frac{70}{3} f_{222}\right) \phi_{0}^{\prime \prime} \\
-2\left(3 f_{4}^{\prime \prime}+10 f_{22}^{\prime \prime}\right) \phi_{2}+7\left(3 f_{4}^{\prime}+10 f_{22}^{\prime}\right) \phi_{2}^{\prime}-5\left(3 f_{4}+10 f_{22}\right) \phi_{2}^{\prime \prime} \\
-20 f_{2}^{\prime \prime} \phi_{4}+35 f_{2}^{\prime} \phi_{4}^{\prime}-15 f_{2} \phi_{4}^{\prime \prime}, \\
\phi_{n}(0)=\phi_{n}^{\prime}(0)=0 \text { and } \phi_{n}^{\prime}(\eta \rightarrow \infty) \rightarrow 1
\end{gathered}
$$

The entire set of BVP's were then solved by a variable step size Runge-Kutta integrator with a relative tolerance of $10^{-6}$. Shooting, using Newton's iteration, was used for the nonlinear equation given by (A.4). 
Equation (A.12) was also solved by a semi-discrete method. Noting that (A.12) is parabolic in $\partial \hat{\chi} / \partial \eta$, the equation was then finite differenced only in the $\zeta$-direction. This then yields a BVP in the $\eta$-direction at each $\zeta_{i}$-location with an additional nonhomogeneous term from the previous step. Using backward differencing, i.e., $\partial \hat{\chi} / \partial \zeta=\left(\hat{\chi}^{i}-\right.$ $\left.\hat{\chi}^{i-1}\right) / \Delta \zeta$, the resulting BVP at each $\zeta_{i}$ is

$$
\left(L_{2} \frac{\partial \hat{\chi}}{\partial \eta}+L_{1} \hat{\chi} / \Delta \zeta\right)_{i}=\left(L_{1}\right)_{i} \hat{\chi}_{i-1} / \Delta \zeta-i \alpha \operatorname{Cos} \zeta-\operatorname{Cos} 2 \zeta
$$

This equation was then solved by a general purpose spline collocation code. The accuracy is effectively controlled by the step size, $\Delta \zeta$, since the relative error tolerance for the $\eta$-integration was $10^{-6}$.

This procedure is a variation of the method of lines concept and has been used by the author on similar unsteady boundary layer problems. Its advantages over a full finite-difference formulation are the enhanced resolution of the unsteadiness in the $\eta$-direction and the fact that the finite difference characteristics approach the continuum characteristics as $\Delta \zeta \rightarrow 0$.

\section{REFERENCES}

[1] Ackerberg, R. C. and Phillips, J. H., The unsteady laminar boundary layer on a semi-infinite plate due to small fluctuations in magnitude of the free-stream velocity, J. Fluid Mech. 51, 137-157 (1972)

[2] Bearman, P. W., Vortex shedding from oscillating bluff bodies, Amer. Rev. Fluid Mech. 16, 195-222 (1984)

[3] Cebeci, T. and Bradshaw, P., Momentum transfer in boundary layers, Hemisphere Pub. Corp., McGraw Hill, 1977

[4] Dwyer, H. A. and McCroskey, W. J., Oscillating flow over a cylinder at large Reynolds number, J. Fluid Mech. 61, 753-767 (1973)

[5] Gersten, Klaus, Higher-order boundary layer theory, Fluid Dyn. Trans. 7, 7-36 (1975)

[6] Ghoshal, S. and Ghoshal, A., Thermal boundary laver theory near the stagnation point in three-dimensional fluctuating flow, J. Fluid Mech. 43, 465-476 (1970)

[7] Glauert, M. B., The laminar boundary layer on oscillating plates and cylinders, J. Fluid Mech. 1, 97-110 (1956)

[8] Ishigaki, H., Periodic boundary layer near a two dimensional stagnation point, J. Fluid Mech. 43, 477-486 (1970)

[9] Lighthill, M. J., The response of laminar skin friction and heat transfer to fluctuations in the stream velocity, Proc. Royal Society 224A, 1-23 (1954)

[10] Lin, C. C., Motion in the boundary layer with a rapidly oscillating external flow, Proc. 9th Int. Congress Applied Mechanics 4, 155-169 (1956)

[11] McCroskey, W. J., Some current research in unsteady fluid dynamics, ASEE J. Fluids Engr. 99, 8-33 (1977)

[12] Riley, N., Unsteady laminar boundary layers, SIAM Review 17, 274-297 (1975)

[13] Rott, N., Unsteady viscous flow in the vicinity of a stagnation point, Quart. Appl. Math. 13, 444-451 (1956)

[14] Stuart, J. T., Double boundary layers in oscillatory viscous flow, J. Fluid Mech. 24, 673-687 (1966)

[15] Van Dyke, M., Higher approximations in boundary laver theory, Annual Review of Fluid Mech. 1, 265-292 (1969) 